\title{
Origin-independent calculation of
}

\section{quadrupole intensities in X-ray spectroscopy}

Stephan Bernadotte, Andrew J. Atkins, and Christoph R. Jacob ${ }^{1}$,

Karlsruhe Institute of Technology (KIT),

Center for Functional Nanostructures and Institute of Physical Chemistry,

Wolfgang-Gaede-Str. 1a, 76131 Karlsruhe, Germany

$\begin{array}{ll}\text { Date: } & \text { November 27, } 2012 \\ \text { Status: } & \text { published in J. Chem. Phys. 137, 204106 (2012). } \\ \text { DOI: } & \text { http://dx.doi.org/10.1063/1.4766359 }\end{array}$

${ }^{1}$ E-Mail: christoph.jacob@kit.edu 


\begin{abstract}
For electronic excitations in the ultraviolet and visible range of the electromagnetic spectrum, the intensities are usually calculated within the dipole approximation, which assumes that the oscillating electric field is constant over the length scale of the transition. For the short wavelengths used in hard X-ray spectroscopy, the dipole approximation may not be adequate. In particular, for metal K-edge X-ray absorption spectroscopy (XAS), it becomes necessary to include higher-order contributions. In quantum-chemical approaches to X-ray spectroscopy, these socalled quadrupole intensities have so far been calculated by including contributions depending on the square of the electric-quadrupole and magnetic-dipole transition moments. However, the resulting quadrupole intensities depend on the choice of the origin of the coordinate system. Here, we show that for obtaining an originindependent theory, one has to include all contributions that are of the same order in the wave vector consistently. This leads to two additional contributions depending on products of the electric-dipole andelectric-octupole and of the electric-dipole and magnetic-quadrupole transition moments, respectively. We have implemented such an origin-independent calculation of quadrupole intensities in XAS within timedependent density-functional theory, and demonstrate its usefulness for the calculation of metal and ligand K-edge XAS spectra of transition metal complexes.
\end{abstract}




\section{Introduction}

X-ray spectroscopy $[1-3]$ is a powerful spectroscopic tool for the elucidation of structural and electronic properties of materials [4 6$]$ and (bio-)molecular systems [7] absorption spectroscopy (XAS) probes the excitation of core electrons. Here, one distinguishes excitations to low-lying unoccupied states (so-called prepeaks), excitations to states close to the ionization threshold (X-ray absorption near-edge structure, XANES), and excitations to continuum states (extended x-ray absorption fine structure, EXAFS). On the other hand, X-ray emission spectroscopy (XES) considers the emission of X-ray radiation after the formation of a core hole.

Of particular interest are the applications of these techniques to study catalytic mechanisms in situ (for examples, see, Ref. [3, 7, 10, 11]) and to investigate biological or biomimetic systems containing transition metal centers (see, e.g., Refs. 12 18]). Usually, XAS and XES are used as fingerprint techniques in such studies to identify the oxidation state, spin state, and local coordination environment of a metal center. This requires either the comparison with spectra measured for model compounds or with theoretical predictions. To be able to extract additional information from X-ray spectroscopic measurements, the development of theoretical methods for the calculation of X-ray spectra is essential [19].

For EXAFS spectra, approaches based on scattering theory are well established and make it possible to extract structural parameters such as distances and coordination numbers 20,21. In contrast, for describing prepeaks and the XANES region in XAS spectroscopy and for predicting XES spectra, quantum-chemical approaches are usually required. To this end, a wide range of quantum-chemical methods have been developed for describing excitations from core orbitals (for reviews, see, e.g., Refs $19,22,23$ ). Widely used are the static-exchange approximation (STEX) 24 26], approaches based on tran- 
sition potential density-functional theory (DFT) [27, 28], and time-dependent densityfunctional theory (TD-DFT) 23]. Within TD-DFT, core excitations are accessible by restricting the space of occupied-virtual orbital excitations (restricted-channel approximation) [29, 30], by selectively targeting excitations within a specific energy window [31 34 , by using a complex polarization propagator [35, 36], or with real-time TD-DFT methods [37]. Recently, coupled-cluster response theory has also been extended to X-ray spectroscopy 38,39$]$.

By combining X-ray spectroscopy with quantum-chemical calculations, it becomes possible to extract information on the electronic structure of molecular systems. For instance, the analysis of the prepeak intensities in ligand K-edge XAS spectra of transition metal complexes (i.e., excitations from the ligand $1 s$ to metal $d$ orbitals) provides insights into the covalent contributions to metal-ligand bonding [40 46]. Another example is metal K-edge XAS, probing excitations from metal $1 s$ to $d$ orbitals, which can be used to assign coordination numbers 47,49 and to probe details of the metal-ligand bonding mechanisms [18, 44, 50]. Recently, we have demonstrated that the prepeaks in Fe K-edge XAS spectra of ferrocene derivatives are sensitive to subtle differences in the electronic structure at the iron atom, which are induced by substituents at the cyclopentadienyl rings, i.e., beyond the first coordination shell of the metal center [51]. Such studies are facilitated by high-energy resolution fluorescence detection (HERFD) techniques, which can resolve the prepeaks with a much higher resolution than conventional XAS measurements [52 54].

However, beside the challenges posed by the accurate quantum-chemical prediction of the absolute or relative energies of core excitations, for the prepeaks in K-edge XAS spectra, theoretical X-ray spectroscopy faces an additional problem. For the calculation of XAS intensities for hard X-rays, the well-known dipole approximation, in which the oscillator strengths are proportional to the square of the electric-dipole transition moments is not sufficient. The dipole approximation is based on the assumption that the wavelength 
of the electromagnetic radiation is large compared to the size of the core orbital. For the high-energy radiation used in hard X-ray spectroscopy, this is not the case anymore. This is particularly important for the prepeaks in metal K-edge XAS spectra of transition metal complexes, which are usually dipole forbidden or have a very low dipole intensity due to symmetry. Thus, the intensity of these prepeaks is due to contributions that are not included in the dipole approximation 55 57.

Currently, contributions to XAS or XES intensities beyond the dipole approximation, socalled quadrupole intensities, are calculated by including additional contributions that are proportional to the squares of the electric-quadrupole and the magnetic-dipole transition moments [58, 59]. However, these additional contributions depend on the choice of the origin of the coordinate system. This situation is not satisfactory, as a physical observable should be origin independent. To rectify this, Neese and coworkers suggested to choose the origin differently for each excitation such that these additional contributions are minimized [58]. Usually, this is equivalent to placing the origin on the atom at which the excitation takes place. However, in cases where the dipole intensities are very small compared to the quadrupole intensities, this scheme sometimes places the origin far away from the excited core orbital [51], which seems unphysical and affects the resulting intensities significantly. Moreover, the scheme will also fail for excitations from core orbitals that are delocalized over different atomic centers, a situation which occurs for ligand-edge XAS spectra or for metal K-edge spectra in polynuclear transition metal clusters.

Thus, a theoretical framework for the origin-independent calculation of quadrupole intensities in X-ray spectroscopy would be desirable. Here, we show that such a formulation can be obtained if all contributions to the oscillator strengths that are of the same order in the wave vector are included consistently.

This work is organized as follows. The theory is presented in Section 2, After introducing the theoretical framework in Sections 2.1 and 2.2 , we revisit the multipole expansion 
of the transition moments in Section 2.3. Subsequently, in Section 2.4 this expansion is applied for the calculation of the oscillator strengths and we demonstrate that these become origin-independent if all terms that are of the same order are included consistently. The final equations for the isotropically averaged quadrupole intensities are then derived in Section 2.5. This is followed by a description of our implementation of the resulting formalism within TD-DFT in Section 3, before we illustrate its usefulness for two test cases in Section 4. Finally a summary and concluding remarks are given in Sect. 5

\section{Theory}

For the theoretical description of spectroscopic processes, quantum chemistry commonly employs a semi-classical theory. In this framework, the molecules are described with (nonrelativistic) quantum-mechanics, whereas the electromagnetic radiation is treated classically (for a discussion, see also Ref. [60]). This theoretical framework is also appropriate for absorption and emission processes in X-ray spectroscopy. Here, we will focus on the case of absorption, but the results can be transferred to other types of experiments.

\subsection{Electromagnetic Radiation}

Within the Coulomb gauge (i.e., if one chooses the vector potential such that $\boldsymbol{\nabla} \cdot \boldsymbol{A}=0$ ), a monochromatic, linearly-polarized electromagnetic wave is defined by the scalar and vector potentials 61,63 ,

$$
\begin{aligned}
\phi(\boldsymbol{r}, t) & =0 \\
\boldsymbol{A}(\boldsymbol{r}, t) & =-A_{0} \mathcal{E} \cos (\boldsymbol{k} \cdot \boldsymbol{r}-\omega t),
\end{aligned}
$$

where the wave vector $\boldsymbol{k}$ points in the direction of propagation and its magnitude is related to the wavelength by $\lambda=2 \pi / k$, where $k=|\boldsymbol{k}|$. The angular frequency $\omega$ is $\omega=2 \pi \nu$ 
with the frequency $\nu$, and frequency and wavelength are related by $c=\lambda \nu=\omega / k$, where $c$ is the speed of light. Finally, the polarization vector $\mathcal{E}$ is a real unit vector that is perpendicular to the direction of propagation (i.e., $\mathcal{E} \cdot \boldsymbol{k}=0$ ).

From these scalar and vector potentials, one obtains for the electric and magnetic fields,

$$
\begin{aligned}
& \boldsymbol{E}(\boldsymbol{r}, t)=-\nabla \phi(\boldsymbol{r}, t)-\frac{1}{c} \frac{\partial \boldsymbol{A}(\boldsymbol{r}, t)}{\partial t}=A_{0} k \mathcal{E} \sin (\boldsymbol{k} \cdot \boldsymbol{r}-\omega t) \\
& \boldsymbol{B}(\boldsymbol{r}, t)=\boldsymbol{\nabla} \times \boldsymbol{A}(\boldsymbol{r}, t)=A_{0}(\boldsymbol{k} \times \mathcal{E}) \sin (\boldsymbol{k} \cdot \boldsymbol{r}-\omega t)
\end{aligned}
$$

Here and in the following, we are using the Gaussian system of units. The electric and magnetic fields are perpendicular to each other and to the direction of propagation and are oscillating with angular frequency $\omega$ and the wavelength $\lambda$. The amplitudes of the electric and magnetic fields are $E_{0}=B_{0}=A_{0} k$.

The intensity $I(\omega)$ of the electromagnetic radiation is defined as the energy flux per area through a surface perpendicular to the propagation direction. It can be calculated from the Poynting vector [61],

$$
\boldsymbol{S}=\frac{c}{4 \pi}(\boldsymbol{E} \times \boldsymbol{B})
$$

by taking the absolute value and averaging over one period of the oscillations,

$$
I(\omega)=\int_{0}^{1 / \nu}|\boldsymbol{S}| \mathrm{d} t=\frac{1}{8 \pi} \frac{\omega^{2}}{c} A_{0}^{2}=\frac{c}{8 \pi} k^{2} A_{0}^{2} .
$$

\subsection{Molecules in an Electromagnetic Field}

In the absence of an external electromagnetic field, a molecular system within the BornOppenheimer approximation is described by the nonrelativistic Hamiltonian

$$
\hat{H}_{0}=\sum_{i=1}^{N} \frac{\hat{\boldsymbol{p}}_{i}^{2}}{2 m_{e}}+V\left(\boldsymbol{r}_{1}, \ldots, \boldsymbol{r}_{N}\right),
$$

where the momentum operator is given by $\hat{p}=-\mathrm{i} \hbar \nabla, m_{e}$ and $e$ are the mass and the charge of the electron, respectively, and the potential energy $V\left(\boldsymbol{r}_{1}, \ldots, \boldsymbol{r}_{N}\right)$ contains the 
electron-nuclei attraction as well as the electron-electron repulsion. Here and in the following, the index $i$ is used to label the electrons.

An external vector potential can be included in this Hamiltonian via 62 64,

$$
\begin{gathered}
\hat{H}=\sum_{i} \frac{1}{2 m_{e}}\left[\hat{\boldsymbol{p}}_{i}-\frac{e}{c} \boldsymbol{A}\left(\boldsymbol{r}_{i}, t\right)\right]^{2}-\frac{g e}{2 m_{e} c} \sum_{i} \boldsymbol{B}\left(\boldsymbol{r}_{i}, t\right) \cdot \hat{\boldsymbol{s}}_{i}+V\left(\boldsymbol{r}_{1}, \ldots, \boldsymbol{r}_{N}\right) \\
=\sum_{i}\left[\frac{\hat{\boldsymbol{p}}_{i}^{2}}{2 m_{e}}-\frac{e}{m_{e} c} \boldsymbol{A}\left(\boldsymbol{r}_{i}, t\right) \cdot \hat{\boldsymbol{p}}_{i}+\frac{e^{2}}{2 m_{e} c^{2}} \boldsymbol{A}^{2}\left(\boldsymbol{r}_{i}, t\right)\right] \\
-\frac{g e}{2 m_{e} c} \sum_{i} \boldsymbol{B}\left(\boldsymbol{r}_{i}, t\right) \cdot \hat{\boldsymbol{s}}_{i}+V\left(\boldsymbol{r}_{1}, \ldots, \boldsymbol{r}_{N}\right)
\end{gathered}
$$

where $g$ is the electron $g$-factor. In the second line we used that in the Coulomb gauge, $\boldsymbol{p}_{i} \cdot \boldsymbol{A}=\boldsymbol{A} \cdot \boldsymbol{p}_{i}$. After neglecting the term that is quadratic in $\boldsymbol{A}$, which is justified for weak electromagnetic fields, this can be expressed as

$$
\hat{H}=\hat{H}_{0}+\hat{U}(t)
$$

where the time-dependent perturbation is given by

$$
\begin{aligned}
\hat{U}(t) & =-\frac{e}{m_{e} c} \sum_{i} \boldsymbol{A}\left(\boldsymbol{r}_{i}, t\right) \cdot \hat{\boldsymbol{p}}_{i}-\frac{g e}{2 m_{e} c} \sum_{i} \boldsymbol{B}\left(\boldsymbol{r}_{i}, t\right) \cdot \hat{\boldsymbol{s}}_{i} \\
& =\frac{e A_{0}}{m_{e} c} \sum_{i}\left[\cos \left(\boldsymbol{k} \cdot \boldsymbol{r}_{i}-\omega t\right)\left(\mathcal{E} \cdot \hat{\boldsymbol{p}}_{i}\right)-\frac{g}{2} \sin \left(\boldsymbol{k} \cdot \boldsymbol{r}_{i}-\omega t\right)(\boldsymbol{k} \times \mathcal{E}) \cdot \hat{\boldsymbol{s}}_{i}\right] .
\end{aligned}
$$

Here, we inserted the vector potential and the magnetic field of an electromagnetic wave given in Eqs. (2) and (4). Using $\sin (x)=\frac{1}{2 \mathrm{i}}[\exp (\mathrm{i} x)-\exp (-\mathrm{i} x)]$, this can be expressed in the form

$$
\hat{U}(t)=\hat{U} \exp (-\mathrm{i} \omega t)+\hat{U}^{*} \exp (\mathrm{i} \omega t)
$$

with the time-independent perturbation operator,

$$
\hat{U}=\frac{e A_{0}}{2 m_{e} c} \sum_{i}\left[\exp \left(\mathrm{i} \boldsymbol{k} \cdot \boldsymbol{r}_{i}\right)\left(\mathcal{E} \cdot \hat{\boldsymbol{p}}_{i}\right)+\mathrm{i} \frac{g}{2} \exp \left(\mathrm{i} \boldsymbol{k} \cdot \boldsymbol{r}_{i}\right)(\boldsymbol{k} \times \mathcal{E}) \cdot \hat{\boldsymbol{s}}_{i}\right]
$$

With this form of the perturbation, we can apply Fermi's golden rule to obtain the transition rate (i.e., the rate of change in the probability of finding the molecule in the $n$-th 
excited state) 62, 63, 65

$$
\Gamma_{0 n}(\omega)=\frac{2 \pi}{\hbar}|\langle 0|\hat{U}| n\rangle|^{2} \delta\left(\omega-\omega_{0 n}\right)=\frac{\pi A_{0}^{2}}{2 \hbar c^{2}}\left|T_{0 n}\right|^{2} \delta\left(\omega-\omega_{0 n}\right),
$$

where we introduced the transition moments

$$
T_{0 n}=\frac{e}{m_{e}} \sum_{i}\left\langle 0\left|\exp \left(\mathrm{i} \boldsymbol{k} \cdot \boldsymbol{r}_{i}\right)\left(\hat{\boldsymbol{p}}_{i} \cdot \mathcal{E}\right)+\mathrm{i} \frac{g}{2} \exp \left(\mathrm{i} \boldsymbol{k} \cdot \boldsymbol{r}_{i}\right)(\boldsymbol{k} \times \mathcal{E}) \cdot \hat{\boldsymbol{s}}_{i}\right| n\right\rangle .
$$

Here, $|0\rangle$ and $|n\rangle$ are the eigenfunctions of the time-independent Hamiltonian $\hat{H}_{0}$ with $\hat{H}_{0}|n\rangle=E_{n}|n\rangle$, and transitions only occur if the frequency of the perturbation matches the energy differences between eigenstates of the unperturbed molecule, i.e., for $\omega=\omega_{0 n}=$ $\left(E_{n}-E_{0}\right) / \hbar$.

Now, Eq. (6) can be used to eliminate $A_{0}^{2}$ from the equation for the transition rate to arrive at,

$$
\Gamma_{0 n}(\omega)=\frac{4 \pi^{2}}{c \hbar \omega^{2}}\left|T_{0 n}\right|^{2} I(\omega) \delta\left(\omega-\omega_{0 n}\right) .
$$

The absorption cross section, describing the rate of energy transfer from the electromagnetic radiation to the molecule, is defined as

$$
\sigma_{0 n}=\int \frac{\Gamma_{0 n}(\omega) \hbar \omega}{I(\omega)} \mathrm{d} \omega=\frac{4 \pi^{2} \hbar}{c E_{0 n}}\left|T_{0 n}\right|^{2}
$$

where $E_{0 n}=E_{n}-E_{0}$. Finally, one usually introduces the dimensionless oscillator strengths,

$$
f_{0 n}=\frac{m_{e} c}{2 \pi^{2} e^{2} \hbar} \sigma_{0 n}=\frac{2 m_{e}}{e^{2} E_{0 n}}\left|T_{0 n}\right|^{2} .
$$

These are defined as transition rates relative to a harmonic oscillator model [63, 65], which fixes the prefactor connecting the absorption cross section and the oscillator strengths.

\subsection{Multipole Expansion}

Calculating the oscillator strengths via the matrix elements of Eq. 14 would in principle be possible, but is cumbersome and in general not feasible. The required integrals are 
difficult to compute analytically (for a possible approach, see Ref. [66]), and because of its dependence on the wave vector $\boldsymbol{k}$, the operator in $T_{0 n}$ is different for each excitation. Therefore, one usually performs a multipole expansion. The starting point for this expansion is a development of the exponential in a Taylor series,

$$
\exp \left(\mathrm{i} \boldsymbol{k} \cdot \boldsymbol{r}_{i}\right)=1+\mathrm{i}\left(\boldsymbol{k} \cdot \boldsymbol{r}_{i}\right)-\frac{1}{2}\left(\boldsymbol{k} \cdot \boldsymbol{r}_{i}\right)^{2}+\cdots
$$

This is substituted into Eq. (14) and, subsequently, one collects the terms of different orders in the wave vector $\boldsymbol{k}$, i.e.,

$$
T_{0 n}=T_{0 n}^{(0)}+T_{0 n}^{(1)}+T_{0 n}^{(2)}+\cdots
$$

In the following, we will consider terms up to second order in $\boldsymbol{k}$. Here, $|\boldsymbol{k}|=2 \pi / \lambda$ acts as the expansion parameter, and we note that for larger wavelengths $\lambda$, the convergence of the Taylor expansion will be faster. For typical molecules and wavelengths in the ultraviolet or visible range, the wavelength is large compared to the molecular size, and it is sufficient to include only the first (zeroth-order) term in this expansion. This corresponds to assuming that the oscillating electric field is constant over the whole molecule. However, for the short wavelengths used in hard X-ray spectroscopy this approximation is not adequate and higher-order terms need to be included.

\subsubsection{Zeroth order: Electric-dipole moment}

In zeroth order in the wave vector $\boldsymbol{k}$, we have

$$
T_{0 n}^{(0)}=\frac{e}{m_{e}} \sum_{i}\left\langle 0\left|\hat{\boldsymbol{p}}_{i} \cdot \mathcal{E}\right| n\right\rangle=\mathcal{E} \cdot\left\langle 0\left|\hat{\boldsymbol{\mu}}^{p}\right| n\right\rangle,
$$

where we have introduced the electric-dipole moment operator in the velocity representation

$$
\hat{\boldsymbol{\mu}}^{p}=\frac{e}{m_{e}} \sum_{i} \hat{\boldsymbol{p}}_{i}
$$


By using the relations given in Appendix A, the matrix elements of the electric-dipole moment operator in the velocity representation can be related to those in the conventional length representation as

$$
\left\langle 0\left|\hat{\boldsymbol{\mu}}^{p}\right| n\right\rangle=-\frac{\mathrm{i}}{\hbar} E_{0 n}\langle 0|\hat{\boldsymbol{\mu}}| n\rangle,
$$

where we introduced the electric-dipole moment operator in the length representation

$$
\hat{\boldsymbol{\mu}}=e \sum_{i} \hat{\boldsymbol{r}}_{i}
$$

Thus, for the zeroth-order contribution, we arrive at

$$
T_{0 n}^{(0)}=T_{0 n}^{(\mu)}=-\mathrm{i} \frac{E_{0 n}}{\hbar}(\mathcal{E} \cdot\langle 0|\hat{\boldsymbol{\mu}}| n\rangle) .
$$

\subsubsection{First order: Electric-quadrupole and magnetic-dipole moments}

In the first order in $\boldsymbol{k}$, we find

$$
T_{0 n}^{(1)}=\frac{\mathrm{i} e}{m_{e}} \sum_{i}\left\langle 0\left|\left(\boldsymbol{k} \cdot \boldsymbol{r}_{i}\right)\left(\hat{\boldsymbol{p}}_{i} \cdot \mathcal{E}\right)\right| n\right\rangle+\frac{\mathrm{i} e g}{2 m_{e}} \sum_{i}\left\langle 0\left|(\boldsymbol{k} \times \mathcal{E}) \cdot \hat{\boldsymbol{s}}_{i}\right| n\right\rangle .
$$

The matrix elements in the first term can be split into one term that is symmetric and one that is antisymmetric with respect to interchanging the wave vector $\boldsymbol{k}$ and the polarization vector $\mathcal{E}$ via

$$
\begin{aligned}
\left\langle 0\left|\left(\boldsymbol{k} \cdot \boldsymbol{r}_{i}\right)\left(\hat{\boldsymbol{p}}_{i} \cdot \mathcal{E}\right)\right| n\right\rangle= & \frac{1}{2}\left\langle 0\left|\left(\boldsymbol{k} \cdot \boldsymbol{r}_{i}\right)\left(\hat{\boldsymbol{p}}_{i} \cdot \mathcal{E}\right)+\left(\boldsymbol{k} \cdot \hat{\boldsymbol{p}}_{i}\right)\left(\boldsymbol{r}_{i} \cdot \mathcal{E}\right)\right| n\right\rangle \\
& +\frac{1}{2}\left\langle 0\left|\left(\boldsymbol{k} \cdot \boldsymbol{r}_{i}\right)\left(\hat{\boldsymbol{p}}_{i} \cdot \mathcal{E}\right)-\left(\boldsymbol{k} \cdot \hat{\boldsymbol{p}}_{i}\right)\left(\boldsymbol{r}_{i} \cdot \mathcal{E}\right)\right| n\right\rangle .
\end{aligned}
$$

From the symmetric first term and using Einstein's convention of implicit summation over

repeated Greek indices, which we use to label the Cartesian components $x, y$, and $z$, we obtain

$$
T_{0 n}^{(Q)}=\frac{\mathrm{i} e}{2 m_{e}} \sum_{i} k_{\alpha} \mathcal{E}_{\beta}\left\langle 0\left|r_{i, \alpha} \hat{p}_{i, \beta}+\hat{p}_{i, \alpha} r_{i, \beta}\right| n\right\rangle=\frac{\mathrm{i}}{2} k_{\alpha} \mathcal{E}_{\beta}\left\langle 0\left|\hat{Q}_{\alpha \beta}^{p}\right| n\right\rangle
$$


where we introduced the electric-quadrupole moment operator in the velocity representation

$$
\hat{Q}_{\alpha \beta}^{p}=\frac{e}{m_{e}} \sum_{i}\left(r_{i, \alpha} \hat{p}_{i, \beta}+\hat{p}_{i, \alpha} r_{i, \beta}\right) .
$$

Again, with the help of the relations given in Appendix A, the matrix elements in the velocity representation can be related to those in the conventional length representation, and one arrives at

$$
T_{0 n}^{(Q)}=\frac{E_{0 n}}{2 \hbar} k_{\alpha} \mathcal{E}_{\beta}\left\langle 0\left|\hat{Q}_{\alpha \beta}\right| n\right\rangle,
$$

where

$$
\hat{Q}_{\alpha \beta}=e \sum_{i} r_{i, \alpha} r_{i, \beta}
$$

is the operator of the electric-quadrupole moment in the length representation. Note that, in contrast to most other authors [58, 67], we do not introduce a traceless version of the quadrupole tensor here. The traceless definition arises from the expansion of $1 /|\boldsymbol{r}|$ that is often introduced in the context of intermolecular interactions, whereas in the case of an expansion of the exponential $\exp (\mathrm{i} \boldsymbol{k} \cdot \boldsymbol{r})$ considered here the definition of Eq. 30 is more natural. Nevertheless, because the wave vector $\boldsymbol{k}$ and the polarization vector $\mathcal{E}$ are orthogonal, the diagonal elements of the electric-quadrupole transition moments do not enter here and it would, therefore, be possible to alter their trace without consequences.

For the antisymmetric second term, we can use that $\left(\boldsymbol{k} \cdot \boldsymbol{r}_{i}\right)$ and $\left(\hat{\boldsymbol{p}}_{i} \cdot \mathcal{E}\right)$ commute because $\boldsymbol{k}$ and $\mathcal{E}$ are orthogonal and then apply the vector identity

$$
(\boldsymbol{a} \cdot \boldsymbol{c})(\boldsymbol{b} \cdot \boldsymbol{d})-(\boldsymbol{b} \cdot \boldsymbol{c})(\boldsymbol{a} \cdot \boldsymbol{d})=(\boldsymbol{a} \times \boldsymbol{b})(\boldsymbol{c} \times \boldsymbol{d})
$$

to obtain,

$$
\begin{aligned}
T_{0 n}^{\left(m^{\prime}\right)} & =\frac{\mathrm{i} e}{2 m_{e}} \sum_{i}\left\langle 0\left|\left(\boldsymbol{k} \cdot \boldsymbol{r}_{i}\right)\left(\hat{\boldsymbol{p}}_{i} \cdot \mathcal{E}\right)-\left(\boldsymbol{k} \cdot \hat{\boldsymbol{p}}_{i}\right)\left(\boldsymbol{r}_{i} \cdot \mathcal{E}\right)\right| n\right\rangle \\
& =\mathrm{i} \frac{e}{2 m_{e}} \sum_{i}\left\langle 0\left|(\boldsymbol{k} \times \mathcal{E})\left(\boldsymbol{r}_{i} \times \hat{\boldsymbol{p}}_{i}\right)\right| n\right\rangle=\mathrm{i} c(\boldsymbol{k} \times \mathcal{E}) \cdot\left\langle 0\left|\hat{\boldsymbol{m}}^{\prime}\right| n\right\rangle,
\end{aligned}
$$


with the (spin-independent) orbital magnetic-dipole moment operator

$$
\hat{\boldsymbol{m}}^{\prime}=\frac{e}{2 m_{e} c} \sum_{i}\left(\boldsymbol{r}_{i} \times \hat{\boldsymbol{p}}_{i}\right)
$$

Thus, this antisymmetric term $T_{0 n}^{\left(m^{\prime}\right)}$ adopts the same form as the last, spin-dependent term in Eq. (25),

$$
T_{0 n}^{\left(m^{s}\right)}=\mathrm{i} \frac{e}{2 m_{e}} \sum_{i}(\boldsymbol{k} \times \mathcal{E}) \cdot\left\langle 0\left|g \hat{\boldsymbol{s}}_{i}\right| n\right\rangle=\mathrm{i} c(\boldsymbol{k} \times \mathcal{E}) \cdot\left\langle 0\left|\hat{\boldsymbol{m}}^{s}\right| n\right\rangle,
$$

with the spin magnetic-dipole operator

$$
\hat{\boldsymbol{m}}^{s}=\frac{e}{2 m_{e} c} \sum_{i} g \hat{\boldsymbol{s}}_{i}
$$

Combining the two contributions, we arrive at the magnetic-dipole transition moment,

$$
T_{0 n}^{(m)}=\mathrm{i} \frac{e}{2 m_{e}} \sum_{i}(\boldsymbol{k} \times \mathcal{E}) \cdot\left\langle 0\left|\left(\boldsymbol{r}_{i} \times \hat{\boldsymbol{p}}_{i}\right)+g \hat{\boldsymbol{s}}_{i}\right| n\right\rangle=\mathrm{i} c(\boldsymbol{k} \times \mathcal{E}) \cdot\langle 0|\hat{\boldsymbol{m}}| n\rangle,
$$

and the total magnetic-dipole moment operator,

$$
\hat{\boldsymbol{m}}=\frac{e}{2 m_{e} c} \sum_{i}\left[\left(\boldsymbol{r}_{i} \times \hat{\boldsymbol{p}}_{i}\right)+g \hat{\boldsymbol{s}}_{i}\right] .
$$

Altogether, the first-order transition moments consist of an electric-quadrupole and a magnetic-dipole contribution, i.e.,

$$
T_{0 n}^{(1)}=T_{0 n}^{(Q)}+T_{0 n}^{\left(m^{\prime}\right)}+T_{0 n}^{\left(m^{s}\right)}=T_{0 n}^{(Q)}+T_{0 n}^{(m)}
$$

\subsubsection{Second order: Electric-octupole and magnetic-quadrupole moments}

In second order in $\boldsymbol{k}$, we find,

$$
T_{0 n}^{(2)}=-\frac{e}{2 m_{e}} \sum_{i}\left\langle 0\left|\left(\boldsymbol{k} \cdot \boldsymbol{r}_{i}\right)\left(\boldsymbol{k} \cdot \boldsymbol{r}_{i}\right)\left(\hat{\boldsymbol{p}}_{i} \cdot \mathcal{E}\right)\right| n\right\rangle-\frac{e g}{2 m_{e}} \sum_{i}\left\langle 0\left|\left(\boldsymbol{k} \cdot \boldsymbol{r}_{i}\right)(\boldsymbol{k} \times \mathcal{E}) \cdot \hat{\boldsymbol{s}}_{i}\right| n\right\rangle .
$$

In a similar fashion as for the first-order term above, the matrix elements in the first term are split into a part that is symmetric and one that is antisymmetric with respect 
to interchanging the polarization vector $\mathcal{E}$ with one of the wave vectors $\boldsymbol{k}$,

$$
\begin{aligned}
& \left\langle 0\left|\left(\boldsymbol{k} \cdot \boldsymbol{r}_{i}\right)\left(\boldsymbol{k} \cdot \boldsymbol{r}_{i}\right)\left(\hat{\boldsymbol{p}}_{i} \cdot \mathcal{E}\right)\right| n\right\rangle \\
& =\frac{1}{3}\langle 0|\left(\boldsymbol{k} \cdot \boldsymbol{r}_{i}\right)\left(\boldsymbol{k} \cdot \boldsymbol{r}_{i}\right)\left(\hat{\boldsymbol{p}}_{i} \cdot \mathcal{E}\right) \\
& \quad+\left(\boldsymbol{k} \cdot \boldsymbol{r}_{i}\right)\left(\boldsymbol{k} \cdot \hat{\boldsymbol{p}}_{i}\right)\left(\boldsymbol{r}_{i} \cdot \mathcal{E}\right)+\left(\boldsymbol{k} \cdot \hat{\boldsymbol{p}}_{i}\right)\left(\boldsymbol{k} \cdot \boldsymbol{r}_{i}\right)\left(\boldsymbol{r}_{i} \cdot \mathcal{E}\right)|n\rangle \\
& \quad+\frac{1}{3}\langle 0| 2\left(\boldsymbol{k} \cdot \boldsymbol{r}_{i}\right)\left(\boldsymbol{k} \cdot \boldsymbol{r}_{i}\right)\left(\hat{\boldsymbol{p}}_{i} \cdot \mathcal{E}\right) \\
& \quad-\left(\boldsymbol{k} \cdot \boldsymbol{r}_{i}\right)\left(\boldsymbol{k} \cdot \hat{\boldsymbol{p}}_{i}\right)\left(\boldsymbol{r}_{i} \cdot \mathcal{E}\right)-\left(\boldsymbol{k} \cdot \hat{\boldsymbol{p}}_{i}\right)\left(\boldsymbol{k} \cdot \boldsymbol{r}_{i}\right)\left(\boldsymbol{r}_{i} \cdot \mathcal{E}\right)|n\rangle .
\end{aligned}
$$

For the symmetric first term, we obtain

$$
\begin{aligned}
T_{0 n}^{(O)} & =-\frac{e}{6 m_{e}} \sum_{i} k_{\alpha} k_{\beta} \mathcal{E}_{\gamma}\left\langle 0\left|r_{i, \alpha} r_{i, \beta} \hat{p}_{i, \gamma}+r_{i, \alpha} \hat{p}_{i, \beta} r_{i, \gamma}+\hat{p}_{i, \alpha} r_{i, \beta} r_{i, \gamma}\right| n\right\rangle \\
& =-\frac{1}{6} \sum_{i} k_{\alpha} k_{\beta} \mathcal{E}_{\gamma}\left\langle 0\left|\hat{O}_{\alpha \beta \gamma}^{p}\right| n\right\rangle,
\end{aligned}
$$

with the operator of the electric-octupole moment in velocity representation

$$
\hat{O}_{\alpha \beta \gamma}^{p}=\frac{e}{m_{e}} \sum_{i}\left(r_{i, \alpha} r_{i, \beta} \hat{p}_{i, \gamma}+r_{i, \alpha} \hat{p}_{i, \beta} r_{i, \gamma}+\hat{p}_{i, \alpha} r_{i, \beta} r_{i, \gamma}\right)
$$

Using the relations given in Appendix A, these matrix elements in the velocity representation can be converted to those in the conventional length representation,

$$
T_{0 n}^{(O)}=\mathrm{i} \frac{E_{0 n}}{6 \hbar} k_{\alpha} k_{\beta} \mathcal{E}_{\gamma}\left\langle 0\left|\hat{O}_{\alpha \beta \gamma}\right| n\right\rangle,
$$

with the octupole operator in length representation given by

$$
\hat{O}_{\alpha \beta \gamma}=e \sum_{i} r_{i, \alpha} r_{i, \beta} r_{i, \gamma}
$$

Again, note that our definition differs from the one given elsewhere [67], as we do not introduce a traceless form here. In fact, for the Cartesian expansion of the exponential $\exp (\mathrm{i} \boldsymbol{k} \cdot \boldsymbol{r})$ it turns out that introducing such a traceless definition here is not possible because only the terms depending on the trace of the octupole moments, $\hat{O}_{\alpha \alpha \beta}$ will contribute to the isotropically-averaged oscillator strengths later on. In contrast, when describing 
intermolecular interactions starting from an expansion of $1 /|\boldsymbol{r}|$, these terms are zero and do not appear.

After some algebra (see Appendix B), the antisymmetric part of Eq. (39) can be expressed as

$$
\begin{aligned}
\left.T_{0 n}^{(\mathcal{M}}{ }^{\prime}\right) & =-\frac{e}{6 m_{e}} \sum_{i}(\boldsymbol{k} \times \mathcal{E}) \cdot\left\langle 0\left|\left(\boldsymbol{k} \cdot \boldsymbol{r}_{i}\right) \cdot\left(\boldsymbol{r}_{i} \times \hat{\boldsymbol{p}}_{i}\right)+\left(\boldsymbol{r}_{i} \times \hat{\boldsymbol{p}}_{i}\right)\left(\boldsymbol{k} \cdot \boldsymbol{r}_{i}\right)\right| n\right\rangle \\
& =-\frac{e}{6 m_{e}} \sum_{i}(\boldsymbol{k} \times \mathcal{E})_{\alpha} k_{\beta}\left\langle 0\left|r_{i, \beta}\left(\boldsymbol{r}_{i} \times \hat{\boldsymbol{p}}_{i}\right)_{\alpha}+\left(\boldsymbol{r}_{i} \times \hat{\boldsymbol{p}}_{i}\right)_{\alpha} r_{i, \beta}\right| n\right\rangle \\
& =-\frac{c}{2}(\boldsymbol{k} \times \mathcal{E})_{\alpha} k_{\beta}\left\langle 0\left|\hat{\mathcal{M}}_{\alpha \beta}^{\prime}\right| n\right\rangle,
\end{aligned}
$$

with the (spin-independent) orbital magnetic-quadrupole operator 67, 68,

$$
\hat{\mathcal{M}}_{\alpha \beta}^{\prime}=\frac{e}{2 m_{e} c} \sum_{i} \frac{2}{3}\left(r_{i, \beta}\left(\boldsymbol{r}_{i} \times \hat{\boldsymbol{p}}_{i}\right)_{\alpha}+\left(\boldsymbol{r}_{i} \times \hat{\boldsymbol{p}}_{i}\right)_{\alpha} r_{i, \beta}\right) .
$$

Note that this operator is not symmetric with respect to interchanging $\alpha$ and $\beta$.

The remaining spin-dependent part of Eq. (39) is given by

$$
\begin{aligned}
T_{0 n}^{\left(\mathcal{M}^{s}\right)} & =-\frac{e g}{2 m_{e}} \sum_{i}(\boldsymbol{k} \times \mathcal{E}) \cdot\left\langle 0\left|\left(\boldsymbol{k} \cdot \boldsymbol{r}_{i}\right) \cdot \hat{\boldsymbol{s}}_{i}\right| n\right\rangle \\
& =-\frac{c}{2} \sum_{i}(\boldsymbol{k} \times \mathcal{E})_{\alpha} k_{\beta}\left\langle 0\left|\hat{\mathcal{M}}_{\alpha \beta}^{s}\right| n\right\rangle
\end{aligned}
$$

with the spin contribution to the magnetic-quadrupole operator

$$
\hat{\mathcal{M}}_{\alpha \beta}^{s}=\frac{e}{2 m_{e} c} \sum_{i} g\left(r_{i, \beta} \hat{s}_{i, \alpha}\right) .
$$

Finally, the full magnetic-quadrupole contribution is obtained by adding the orbital and spin contributions to obtain

$$
T_{0 n}^{(\mathcal{M})}=T_{0 n}^{\left(\mathcal{M}^{\prime}\right)}+T_{0 n}^{\left(\mathcal{M}^{s}\right)}=-\frac{c}{2}(\boldsymbol{k} \times \mathcal{E})_{\alpha} k_{\beta}\left\langle 0\left|\hat{\mathcal{M}}^{\prime}{ }_{\alpha \beta}+\hat{\mathcal{M}}_{\alpha \beta}^{s}\right| n\right\rangle .
$$

Altogether, the second-order transition moments consist of an electric-octupole and a magnetic-quadrupole contribution, i.e.,

$$
T_{0 n}^{(2)}=T_{0 n}^{(O)}+T_{0 n}^{\left(\mathcal{M}^{\prime}\right)}+T_{0 n}^{\left(\mathcal{M}^{s}\right)}=T_{0 n}^{(O)}+T_{0 n}^{(\mathcal{M})} .
$$




\subsubsection{Summary of the multipole transition moments}

In summary, the multipole expansion of the transition moments of Eq. (14) up to second order results in five different contributions,

$$
T_{0 n}=T_{0 n}^{(\mu)}+T_{0 n}^{(Q)}+T_{0 n}^{(m)}+T_{0 n}^{(O)}+T_{0 n}^{(\mathcal{M})}+\cdots
$$

The expressions for these contributions are summarized in Table II. In zeroth order, one encounters the well-known electric-dipole transition moments. Starting from first order, an electric and a magnetic contribution appear. In first order, these are the electricquadrupole and magnetic-dipole transition moments, whereas in second order one has the electric-octupole and magnetic-quadrupole transition moments.

[Table 1 about here.]

If we restrict our considerations to a spin-orbit coupling free framework in the absence of static external magnetic fields, the wavefunction can always be chosen as a real function. In this case, the electric transition integrals, $\left\langle 0\left|\hat{\mu}_{\alpha}\right| n\right\rangle,\left\langle 0\left|\hat{Q}_{\alpha \beta}\right| n\right\rangle$, and $\left\langle 0\left|\hat{O}_{\alpha \beta \gamma}\right| n\right\rangle$, as defined here are always real. Moreover, for the magnetic transition moments, the spin contributions can be neglected because for states with a multiplicity larger than zero (i.e., for $S>0$ ) the different $M_{S^{-}}$components of the multiplet will be degenerate 69, 70 and the components with $+M_{S}$ and $-M_{S}$ provide spin contributions to the magnetic transition moments that cancel each other. Therefore, these spin contributions, $\left\langle 0\left|\hat{m}_{\alpha}^{s}\right| n\right\rangle$ and $\left\langle 0\left|\hat{\mathcal{M}}_{\alpha \beta}^{s}\right| n\right\rangle$, will not be considered further in the following. The remaining orbital contribution to the magnetic transition integrals, $\left\langle 0\left|\hat{m}_{\alpha}\right| n\right\rangle$ and $\left\langle 0\left|\hat{\mathcal{M}}_{\alpha \beta}\right| n\right\rangle$, are then purely imaginary. As a consequence, we notice that the zeroth-order and second-order transition moments are purely imaginary, whereas the first-order transition moments are purely real. This holds both for the electric and for the magnetic contributions. 


\subsubsection{Origin dependence of the multipole transition moments}

While the electric-dipole transition moments are independent of the choice of the origin of the coordinate systems, the higher-order transition moments are origin dependent [67]. We give derivations of the expressions for the change of the electric-quadrupole and electicoctupole transition moments and of the magnetic-dipole and magnetic-quadrupole transition moments upon shifting the origin from $\boldsymbol{O}$ to $\boldsymbol{O}+\boldsymbol{a}$ in the Supplementary Material [71]. Here, we only present the final results as required for the following discussions.

For the electric-quadrupole transition moments, one has

$$
\left\langle 0\left|\hat{Q}_{\alpha \beta}(\boldsymbol{O}+\boldsymbol{a})\right| n\right\rangle=\left\langle 0\left|\hat{Q}_{\alpha \beta}(\boldsymbol{O})\right| n\right\rangle-a_{\beta}\left\langle 0\left|\hat{\mu}_{\alpha}\right| n\right\rangle-a_{\alpha}\left\langle 0\left|\hat{\mu}_{\beta}\right| n\right\rangle,
$$

whereas for the electric-octupole transition moments, the corresponding expression becomes,

$$
\begin{aligned}
\left\langle 0\left|\hat{O}_{\alpha \beta \gamma}(\boldsymbol{O}+\boldsymbol{a})\right| n\right\rangle= & \left\langle 0\left|\hat{O}_{\alpha \beta \gamma}(\boldsymbol{O})\right| n\right\rangle \\
& -a_{\gamma}\left\langle 0\left|\hat{Q}_{\alpha \beta}(\boldsymbol{O})\right| n\right\rangle-a_{\beta}\left\langle 0\left|\hat{Q}_{\alpha \gamma}(\boldsymbol{O})\right| n\right\rangle-a_{\alpha}\left\langle 0\left|\hat{Q}_{\beta \gamma}(\boldsymbol{O})\right| n\right\rangle \\
& +a_{\alpha} a_{\beta}\left\langle 0\left|\hat{\mu}_{\gamma}\right| n\right\rangle+a_{\alpha} a_{\gamma}\left\langle 0\left|\hat{\mu}_{\beta}\right| n\right\rangle+a_{\beta} a_{\gamma}\left\langle 0\left|\hat{\mu}_{\alpha}\right| n\right\rangle .
\end{aligned}
$$

Thus, the electric-quadrupole and octupole moments are only origin independent if all lower-order electric transition moments vanish. The above expressions hold both for the length and for the velocity representation.

For the magnetic-dipole transition moment, a shift of the origin of the coordinate systems results in,

$$
\begin{aligned}
\left\langle 0\left|m_{\alpha}^{\prime}(\boldsymbol{O}+\boldsymbol{a})\right| n\right\rangle & =\left\langle 0\left|m_{\alpha}^{\prime}(\boldsymbol{O})\right| n\right\rangle-\varepsilon_{\alpha \beta \gamma} a_{\beta} \frac{1}{2 c}\left\langle 0\left|\hat{\mu}_{\gamma}^{p}\right| n\right\rangle \\
& =\left\langle 0\left|m_{\alpha}^{\prime}(\boldsymbol{O})\right| n\right\rangle+\frac{\mathrm{i}}{2} \varepsilon_{\alpha \beta \gamma} a_{\beta} \frac{E_{0 n}}{\hbar c}\left\langle 0\left|\hat{\mu}_{\gamma}\right| n\right\rangle,
\end{aligned}
$$

where $\varepsilon_{\alpha \beta \gamma}$ is the Levi-Civita tensor. Thus, a term depending on the electric-dipole moment in the velocity representation arises. This can be converted into the length represen- 
tation using the relation from Appendix A. The origin dependence of the magnetic-dipole transition moments vanishes if the electric-dipole transition moment is zero.

Finally, for the magnetic-quadrupole transition moment the origin dependence is given by 68,72

$$
\begin{aligned}
\left\langle 0\left|\mathcal{M}_{\alpha \beta}^{\prime}(\boldsymbol{O}+\boldsymbol{a})\right| n\right\rangle= & \left\langle 0\left|\mathcal{M}_{\alpha \beta}^{\prime}(\boldsymbol{O})\right| n\right\rangle \\
& -\frac{1}{3 c} \varepsilon_{\alpha \gamma \delta} a_{\gamma}\left\langle 0\left|\hat{Q}_{\beta \delta}^{p}(\boldsymbol{O})\right| n\right\rangle+\frac{2}{3 c} \varepsilon_{\alpha \gamma \delta} a_{\beta} a_{\gamma}\left\langle 0\left|\hat{\mu}_{\delta}^{p}\right| n\right\rangle \\
& +\frac{2}{3} \delta_{\alpha \beta}\left(\boldsymbol{a} \cdot\left\langle 0\left|\boldsymbol{m}^{\prime}(\boldsymbol{O})\right| n\right\rangle\right)-2 a_{\beta}\left\langle 0\left|m_{\alpha}^{\prime}(\boldsymbol{O})\right| n\right\rangle .
\end{aligned}
$$

Again, the electric-dipole and electric-quadrupole transition moments in the velocity representation can be converted to the length representation to finally arrive at

$$
\begin{aligned}
\left\langle 0\left|\mathcal{M}_{\alpha \beta}^{\prime}(\boldsymbol{O}+\boldsymbol{a})\right| n\right\rangle= & \left\langle 0\left|\mathcal{M}_{\alpha \beta}^{\prime}(\boldsymbol{O})\right| n\right\rangle \\
& +\frac{\mathrm{i}}{3} \frac{E_{0 n}}{\hbar c} \varepsilon_{\alpha \gamma \delta} a_{\gamma}\left\langle 0\left|\hat{Q}_{\beta \delta}(\boldsymbol{O})\right| n\right\rangle-\frac{2 \mathrm{i}}{3} \frac{E_{0 n}}{\hbar c} \varepsilon_{\alpha \gamma \delta} a_{\beta} a_{\gamma}\left\langle 0\left|\hat{\mu}_{\delta}\right| n\right\rangle \\
& +\frac{2}{3} \delta_{\alpha \beta}\left(\boldsymbol{a} \cdot\left\langle 0\left|\boldsymbol{m}^{\prime}(\boldsymbol{O})\right| n\right\rangle\right)-2 a_{\beta}\left\langle 0\left|m_{\alpha}^{\prime}(\boldsymbol{O})\right| n\right\rangle .
\end{aligned}
$$

Here, we notice that upon shifting the origin, the magnetic-quadrupole transition moment generates all lower-order contributions, i.e., terms depending on the electric-dipole and electric-quadrupole transition moments as well as on the magnetic-dipole transition moments.

\subsection{Oscillator Strengths}

The multipole expansion of the full transition moments $T_{0 n}$ can now be inserted into Eq. 177 to obtain an expression for calculating the oscillator strengths,

$$
\begin{aligned}
f_{0 n} & =\frac{2 m_{e}}{e^{2} E_{0 n}}\left|T_{0 n}^{(0)}+T_{0 n}^{(1)}+T_{0 n}^{(2)}+\cdots\right|^{2} \\
& =\frac{2 m_{e}}{e^{2} E_{0 n}}\left|T_{0 n}^{(\mu)}+T_{0 n}^{(Q)}+T_{0 n}^{(m)}+T_{0 n}^{(O)}+T_{0 n}^{(\mathcal{M})}+\cdots\right|^{2} .
\end{aligned}
$$


Here, different truncations of the expansion can be employed. In the dipole approximation, only the zeroth-order term is retained, and for the oscillator strengths one arrives at the well-known expression in which the oscillator strengths are proportional to the squared absolute value of the electric-dipole transition moments. This approximation is commonly employed in electronic spectroscopy in the ultraviolet and visible region of the electromagnetic spectrum.

Here, we are interested in cases where the dipole approximation breaks down, such as K-edge XAS spectroscopy of transition metal complexes. In such situations, higher-order terms in the multipole expansion have to be included. In the currently used approximation, the multipole expansion of the transition moments is truncated after the first order, i.e., the oscillator strengths are approximated as 58

$$
f_{0 n} \approx \frac{2 m_{e}}{e^{2} E_{0 n}}\left|T_{0 n}^{(0)}+T_{0 n}^{(1)}\right|^{2}
$$

However, it turns out that the resulting expressions depend on the choice of the origin of the coordinate system (see Ref. [58] for details).

[Figure 1 about here.]

To obtain an origin-independent formulation, we return to Eq. 57) and realize that the squared absolute value results in a sum of products of multipole transition moments. These products are of different orders in the wave vector $\boldsymbol{k}$, as is illustrated in Fig. 1 . Hence, it seems logical to retain all terms up to a given order in $\boldsymbol{k}$ in the expression for the oscillator strengths instead of truncating the multipole expansion of the transition moments. By collecting terms that are of the same order, the oscillator strengths can be expressed as

$$
f_{0 n}=f_{0 n}^{(0)}+f_{0 n}^{(1)}+f_{0 n}^{(2)}+\cdots
$$


where

$$
\begin{aligned}
f_{0 n}^{(0)} & =\frac{2 m_{e}}{e^{2} E_{0 n}}\left|T_{0 n}^{(0)}\right|^{2} \\
f_{0 n}^{(1)} & =\frac{2 m_{e}}{e^{2} E_{0 n}} 2 \operatorname{Re}\left(T_{0 n}^{(0), *} T_{0 n}^{(1)}\right)=0 \\
f_{0 n}^{(2)} & =\frac{2 m_{e}}{e^{2} E_{0 n}}\left[\left|T_{0 n}^{(1)}\right|^{2}+2 \operatorname{Re}\left(T_{0 n}^{(0), *} T_{0 n}^{(2)}\right)\right],
\end{aligned}
$$

where the star denotes complex conjugation. Because $T_{0 n}^{(0)}$ is purely imaginary and $T_{0 n}^{(1)}$ is real, their product is also purely imaginary and the first-order contribution $f_{0 n}^{(1)}$ vanishes. In the following, we will retain all terms up to second order and it turns out that the resulting approximation for the oscillator strengths is independent of the choice of the origin.

\subsubsection{Origin independence of oscillator strengths}

Starting from the definitions of $T_{0 n}^{(0)}, T_{0 n}^{(1)}$, and $T_{0 n}^{(2)}$ in Eqs. 20), (25), and (39), respectively, one can easily see that their origin dependence is given by

$$
\begin{aligned}
& T_{0 n}^{(0)}(\boldsymbol{O}+\boldsymbol{a})=T_{0 n}^{(0)}(\boldsymbol{O}), \\
& T_{0 n}^{(1)}(\boldsymbol{O}+\boldsymbol{a})=T_{0 n}^{(1)}(\boldsymbol{O})+\mathrm{i}(\boldsymbol{k} \cdot \boldsymbol{a}) T_{0 n}^{(0)}(\boldsymbol{O}), \\
& T_{0 n}^{(2)}(\boldsymbol{O}+\boldsymbol{a})=T_{0 n}^{(2)}(\boldsymbol{O})+\mathrm{i}(\boldsymbol{k} \cdot \boldsymbol{a}) T_{0 n}^{(1)}(\boldsymbol{O})-\frac{1}{2}(\boldsymbol{k} \cdot \boldsymbol{a})^{2} T_{0 n}^{(0)}(\boldsymbol{O}) .
\end{aligned}
$$

Therefore, the zeroth-order contribution to the oscillator strengths $f_{0 n}^{(0)}$, i.e., the expression obtained in the dipole approximation, is obviously origin independent. For the secondorder contribution, we have

$$
\begin{aligned}
f_{0 n}^{(2)}(\boldsymbol{O}+\boldsymbol{a})= & \frac{2 m_{e}}{e^{2} E_{0 n}}\left[\left|T_{0 n}^{(1)}(\boldsymbol{O}+\boldsymbol{a})\right|^{2}+2 \operatorname{Re}\left(T_{0 n}^{(0), *}(\boldsymbol{O}) T_{0 n}^{(2)}(\boldsymbol{O}+\boldsymbol{a})\right)\right] \\
=\frac{2 m_{e}}{e^{2} E_{0 n}}\left[\left|T_{0 n}^{(1)}(\boldsymbol{O})\right|^{2}+2 \operatorname{Re}\left(\mathrm{i}(\boldsymbol{k} \cdot \boldsymbol{a}) T_{0 n}^{(0)}(\boldsymbol{O}) T_{0 n}^{(1), *}(\boldsymbol{O})\right)+(\boldsymbol{k} \cdot \boldsymbol{a})^{2}\left|T_{0 n}^{(0)}(\boldsymbol{O})\right|^{2}\right. & \left.\quad+\operatorname{Re}\left(T_{0 n}^{(0), *}(\boldsymbol{O})\left[2 T_{0 n}^{(2)}(\boldsymbol{O})+2 \mathrm{i}(\boldsymbol{k} \cdot \boldsymbol{a}) T_{0 n}^{(1)}(\boldsymbol{O})-(\boldsymbol{k} \cdot \boldsymbol{a})^{2} T_{0 n}^{(0)}(\boldsymbol{O})\right]\right)\right] \\
& \quad \\
= & f_{0 n}^{(2)}(\boldsymbol{O})
\end{aligned}
$$


and find that this contribution is indeed independent of the choice of the origin. In fact, it can be shown that for each order, the higher-order contributions to the oscillator strengths are origin independent if all terms that are of the same order in the wave vector $\boldsymbol{k}$ are included. This is demonstrated in Appendix C.

\subsubsection{Dipole and quadrupole oscillator strengths}

After having established an origin-independent definition of the different approximations to the oscillator strengths, we will now turn to deriving explicit expressions. Considering only the zeroth-order contribution corresponds to the dipole approximation, in which the dipole oscillator strengths are given by

$$
f_{0 n} \approx f_{0 n}^{(0)}=f_{0 n}^{\left(\mu^{2}\right)}=\frac{2 m_{e}}{e^{2} \hbar^{2}} E_{0 n}|\mathcal{E} \cdot\langle 0|\hat{\boldsymbol{\mu}}| n\rangle|^{2}=\frac{2 m_{e}}{e^{2} \hbar^{2}} E_{0 n}\left(\mathcal{E}_{\alpha}\left\langle 0\left|\hat{\mu}_{\alpha}\right| n\right\rangle\right)^{2} .
$$

Since the first-order contributions vanish, the next step to go beyond the dipole approximation is to include all second-order contributions. Thus, the oscillator strengths can be approximated as the sum of the dipole (zeroth-order) oscillator strengths and the quadrupole (second-order) oscillator strengths,

$$
f_{0 n} \approx f_{0 n}^{(0)}+f_{0 n}^{(2)}=\left|T_{0 n}^{(0)}\right|^{2}+\left|T_{0 n}^{(1)}\right|^{2}+2 \operatorname{Re}\left(T_{0 n}^{(0), *} T_{0 n}^{(2)}\right) .
$$

We will refer to this approximation as the quadrupole approximation. For the quadrupole oscillator strengths, we can insert the individual multipole transition moments, and obtain five different terms,

$$
\begin{aligned}
f_{0 n}^{(2)} & =\frac{2 m_{e}}{e^{2} E_{0 n}}\left[\left|T_{0 n}^{(Q)}\right|^{2}+\left|T_{0 n}^{(m)}\right|^{2}+2 \operatorname{Re}\left(T_{0 n}^{(Q), *} T_{0 n}^{(m)}\right)+2 \operatorname{Re}\left(T_{0 n}^{(\mu), *} T_{0 n}^{(O)}\right)+2 \operatorname{Re}\left(T_{0 n}^{(\mu), *} T_{0 n}^{(\mathcal{M})}\right)\right] \\
& =f_{0 n}^{\left(Q^{2}\right)}+f_{0 n}^{\left(m^{2}\right)}+f_{0 n}^{(Q m)}+f_{0 n}^{(\mu O)}+f_{0 n}^{(\mu \mathcal{M})} .
\end{aligned}
$$

First, there are three contributions arising from products of first-order transition moments, an electric-quadrupole-electric-quadrupole contribution,

$$
f_{0 n}^{\left(Q^{2}\right)}=\frac{m_{e}}{2 e^{2} \hbar^{2}} E_{0 n}\left(k_{\alpha} \mathcal{E}_{\beta}\left\langle 0\left|\hat{Q}_{\alpha \beta}\right| n\right\rangle\right)^{2},
$$


a magnetic-dipole-magnetic-dipole contribution,

$$
f_{0 n}^{\left(m^{2}\right)}=\frac{2 m_{e} c^{2}}{e^{2} E_{0 n}}\left|(\boldsymbol{k} \times \mathcal{E})_{\alpha}\left\langle 0\left|\hat{m}_{\alpha}\right| n\right\rangle\right|^{2}=\frac{2 m_{e} c^{2}}{e^{2} E_{0 n}}\left((\boldsymbol{k} \times \mathcal{E})_{\alpha} \operatorname{Im}\left\langle 0\left|\hat{m}_{\alpha}\right| n\right\rangle\right)^{2},
$$

and a cross-term, the electric-quadrupole-magnetic-dipole contribution,

$$
f_{0 n}^{(Q m)}=-\frac{2 m_{e} c}{e^{2} \hbar}\left(k_{\alpha} \mathcal{E}_{\beta}\left\langle 0\left|\hat{Q}_{\alpha \beta}\right| n\right\rangle\right)\left((\boldsymbol{k} \times \mathcal{E})_{\alpha} \operatorname{Im}\left\langle 0\left|\hat{m}_{\alpha}\right| n\right\rangle\right) .
$$

These three contributions have been considered previously in the calculation of the quadrupole oscillator strengths in Ref. [58]. In addition, two additional contributions have to be included in order to collect all terms that are of second order and to arrive at an originindependent approximation. These are the electric-dipole-electric-octupole contribution,

$$
f_{0 n}^{(\mu O)}=-\frac{2 m_{e}}{3 \hbar^{2} e^{2}} E_{0 n}\left(\mathcal{E}_{\alpha}\left\langle 0\left|\hat{\mu}_{\alpha}\right| n\right\rangle\right)\left(k_{\alpha} k_{\beta} \mathcal{E}_{\gamma}\left\langle 0\left|\hat{O}_{\alpha \beta \gamma}\right| n\right\rangle\right)
$$

and the electric-dipole-magnetic-quadrupole contribution,

$$
f_{0 n}^{(\mu \mathcal{M})}=\frac{2 m_{e} c}{e^{2} \hbar}\left(\mathcal{E}_{\alpha}\left\langle 0\left|\hat{\mu}_{\alpha}\right| n\right\rangle\right)\left((\boldsymbol{k} \times \mathcal{E})_{\alpha} k_{\beta} \operatorname{Im}\left\langle 0\left|\hat{\mathcal{M}}_{\alpha \beta}\right| n\right\rangle\right)
$$

Now we choose the wave vector as $\boldsymbol{k}=k \boldsymbol{e}_{x}$ along the $x$-axis and the polarization vector as $\mathcal{E}=\boldsymbol{e}_{y}$ along the $y$-axis. Consequently, $\left(\boldsymbol{e}_{x} \times \mathcal{E}\right)$ becomes the unit vector $\boldsymbol{e}_{z}$ along the $z$-axis. This is no loss of generality, as the molecule can still have an arbitrary orientation in the coordinate system. Using

$$
k=\frac{E_{0 n}}{\hbar c},
$$

the different contributions to the oscillator strengths become

$$
\begin{aligned}
f_{0 n}^{\left(\mu^{2}\right)} & =\frac{2 m_{e}}{e^{2} \hbar^{2}} E_{0 n}\left\langle 0\left|\hat{\mu}_{y}\right| n\right\rangle^{2} \\
f_{0 n}^{\left(Q^{2}\right)} & =\frac{m_{e}}{2 e^{2} \hbar^{4} c^{2}} E_{0 n}^{3}\left\langle 0\left|\hat{Q}_{x y}\right| n\right\rangle^{2} \\
f_{0 n}^{\left(m^{2}\right)} & =\frac{2 m_{e}}{e^{2} \hbar^{2}} E_{0 n}\left[\operatorname{Im}\left\langle 0\left|\hat{m}_{z}\right| n\right\rangle\right]^{2} \\
f_{0 n}^{(Q m)} & =-\frac{2 m_{e}}{e^{2} \hbar^{3} c} E_{0 n}^{2}\left\langle 0\left|\hat{Q}_{x y}\right| n\right\rangle \operatorname{Im}\left\langle 0\left|\hat{m}_{z}\right| n\right\rangle
\end{aligned}
$$




$$
\begin{aligned}
f_{0 n}^{(\mu O)} & =-\frac{2 m_{e}}{3 e^{2} \hbar^{4} c^{2}} E_{0 n}^{3}\left\langle 0\left|\hat{\mu}_{y}\right| n\right\rangle\left\langle 0\left|\hat{O}_{x x y}\right| n\right\rangle \\
f_{0 n}^{(\mu \mathcal{M})} & =\frac{2 m_{e}}{e^{2} \hbar^{3} c} E_{0 n}^{2}\left\langle 0\left|\hat{\mu}_{y}\right| n\right\rangle \operatorname{Im}\left\langle 0\left|\hat{\mathcal{M}}_{z x}\right| n\right\rangle .
\end{aligned}
$$

These oscillator strengths refer to an experimental setup in which the incident radiation has a well-defined polarization and in which the molecules have a fixed orientation with respect to the radiation. Using the expressions for the origin dependence of the different multipole transition moments given in Section 2.3.5, it can be verified that the total second-order oscillator strengths calculated using the above equations are originindependent (see Supplementary Material [71]).

\subsection{Isotropic Averaging}

Often, the molecules are not oriented with respect to the incident radiation in experiments, but the measurement is performed in solution where the molecules can freely rotate. Thus, to arrive at final expressions for the oscillator strengths in such experiments, we have to perform an averaging over all possible orientations of the molecule.

The expressions for performing this averaging are derived, for instance, in Ref. [67] (see in particular chapter 4.2). For the isotropic averages of tensors with two, three, and four Cartesian indices, one finds

$$
\begin{aligned}
\left\langle T_{x x}\right\rangle_{\text {iso }} & =\sum_{\alpha \beta}\left\langle i_{\alpha} i_{\beta}\right\rangle_{\text {iso }} T_{\alpha \beta} \\
\left\langle T_{x y z}\right\rangle_{\text {iso }} & =\sum_{\alpha \beta \gamma}\left\langle i_{\alpha} j_{\beta} k_{\gamma}\right\rangle_{\text {iso }} T_{\alpha \beta \gamma} \\
\left\langle T_{x x y y}\right\rangle_{\text {iso }} & =\sum_{\alpha \beta \gamma \delta}\left\langle i_{\alpha} i_{\beta} j_{\gamma} j_{\delta}\right\rangle_{\text {iso }} T_{\alpha \beta \gamma \delta},
\end{aligned}
$$

where the isotropic averages of the Cartesian unit vector $\boldsymbol{i}=\boldsymbol{e}_{x}, \boldsymbol{j}=\boldsymbol{e}_{y}$, and $\boldsymbol{k}=\boldsymbol{e}_{z}$ are 
given by

$$
\begin{aligned}
\left\langle i_{\alpha} i_{\beta}\right\rangle_{\text {iso }} & =\frac{1}{3} \delta_{\alpha \beta}, \\
\left\langle i_{\alpha} j_{\beta} k_{\gamma}\right\rangle_{\text {iso }} & =\frac{1}{6} \epsilon_{\alpha \beta \gamma}, \\
\left\langle i_{\alpha} i_{\beta} j_{\gamma} j_{\delta}\right\rangle_{\text {iso }} & =\frac{1}{30}\left(4 \delta_{\alpha \beta} \delta_{\gamma \delta}-\delta_{\alpha \gamma} \delta_{\beta \delta}-\delta_{\alpha \delta} \delta_{\beta \gamma}\right) .
\end{aligned}
$$

For all other tensor components, such as, e.g., $\left\langle T_{x y}\right\rangle_{\text {iso }}$ or $\left\langle T_{x x y}\right\rangle_{\text {iso }}$, the isotropic averages are zero.

Using these expressions, we obtain for the isotropically averaged electric-dipole-electricdipole contribution to the oscillator strengths,

$$
\left\langle f_{0 n}^{\left(\mu^{2}\right)}\right\rangle_{\text {iso }}=\frac{2 m_{e}}{3 e^{2} \hbar^{2}} E_{0 n} \sum_{\alpha}\left\langle 0\left|\hat{\mu}_{\alpha}\right| n\right\rangle^{2}=\frac{2 m_{e}}{3 e^{2} \hbar^{2}} E_{0 n}\langle 0|\hat{\boldsymbol{\mu}}| n\rangle^{2}
$$

Similarly, for the electric-quadrupole-electric-quadrupole contribution, we find

$$
\begin{aligned}
\left\langle f_{0 n}^{\left(Q^{2}\right)}\right\rangle_{\text {iso }} & =\frac{m_{e}}{60 e^{2} \hbar^{4} c^{2}} E_{0 n}^{3} \sum_{\alpha \beta \gamma \delta}\left(4 \delta_{\alpha \gamma} \delta_{\beta \delta}-\delta_{\alpha \beta} \delta_{\gamma \delta}-\delta_{\alpha \delta} \delta_{\beta \gamma}\right)\left\langle 0\left|\hat{Q}_{\alpha \beta}\right| n\right\rangle\left\langle 0\left|\hat{Q}_{\gamma \delta}\right| n\right\rangle \\
& =\frac{m_{e}}{20 e^{2} \hbar^{4} c^{2}} E_{0 n}^{3}\left[\sum_{\alpha \beta}\left\langle 0\left|\hat{Q}_{\alpha \beta}\right| n\right\rangle^{2}-\frac{1}{3}\left(\sum_{\alpha}\left\langle 0\left|\hat{Q}_{\alpha \alpha}\right| n\right\rangle\right)^{2}\right] .
\end{aligned}
$$

We note that this is identical to the expression in Ref. [58], where a traceless definition of the quadrupole moment is used. For the magnetic-dipole-magnetic-dipole contribution, the isotropic average is,

$$
\left\langle f_{0 n}^{\left(m^{2}\right)}\right\rangle_{\text {iso }}=\frac{2 m_{e}}{3 e^{2} \hbar^{2}} E_{0 n} \sum_{\alpha} \operatorname{Im}\left\langle 0\left|\hat{m}_{\alpha}\right| n\right\rangle^{2}=\frac{2 m_{e}}{3 e^{2} \hbar^{2}} E_{0 n}(\operatorname{Im}\langle 0|\hat{\boldsymbol{m}}| n\rangle)^{2}
$$

The isotropic average of the electric-quadrupole-magnetic-dipole contribution to the oscillator strengths,

$$
\left\langle f_{0 n}^{(Q m)}\right\rangle_{\text {iso }}=-\frac{m_{e}}{3 e^{2} \hbar^{3} c} E_{0 n}^{2} \sum_{\alpha \beta \gamma} \varepsilon_{\alpha \beta \gamma}\left\langle 0\left|\hat{Q}_{\alpha \beta}\right| n\right\rangle\left\langle 0\left|\hat{m}_{\gamma}\right| n\right\rangle=0
$$


turns out to be zero because $\left\langle 0\left|\hat{Q}_{\alpha \beta}\right| n\right\rangle=\left\langle 0\left|\hat{Q}_{\beta \alpha}\right| n\right\rangle$. Finally, for the electric-dipoleelectric-octupole contribution to the oscillator strengths, we obtain

$$
\begin{aligned}
\left\langle f_{0 n}^{(\mu O)}\right\rangle_{\text {iso }} & =-\frac{m_{e}}{45 e^{2} \hbar^{4} c^{2}} E_{0 n}^{3} \sum_{\alpha \beta \gamma \delta}\left(4 \delta_{\alpha \beta} \delta_{\gamma \delta}-\delta_{\alpha \gamma} \delta_{\beta \delta}-\delta_{\alpha \delta} \delta_{\beta \gamma}\right)\left\langle 0\left|\hat{\mu}_{\delta}\right| n\right\rangle\left\langle 0\left|\hat{O}_{\alpha \beta \gamma}\right| n\right\rangle \\
& =-\frac{2 m_{e}}{45 e^{2} \hbar^{4} c^{2}} E_{0 n}^{3} \sum_{\alpha \beta}\left\langle 0\left|\hat{\mu}_{\beta}\right| n\right\rangle\left\langle 0\left|\hat{O}_{\alpha \alpha \beta}\right| n\right\rangle,
\end{aligned}
$$

where we used the symmetry of the octupole moments with respect to the exchange of indices, and for the electric-dipole-magnetic-quadrupole contribution,

$$
\left\langle f_{0 n}^{(\mu \mathcal{M})}\right\rangle_{\text {iso }}=\frac{m_{e}}{3 e^{2} \hbar^{3} c} E_{0 n}^{2} \sum_{\alpha \beta \gamma} \varepsilon_{\alpha \beta \gamma}\left\langle 0\left|\hat{\mu}_{\beta}\right| n\right\rangle \operatorname{Im}\left\langle 0\left|\hat{\mathcal{M}}_{\gamma \alpha}\right| n\right\rangle .
$$

Note again that the magnetic-quadrupole transition moments are in general not symmetric or antisymmetric with respect to the interchange of the two Cartesian indices, i.e., $\left\langle 0\left|\mathcal{M}_{\alpha \beta}\right| n\right\rangle \neq \pm\left\langle 0\left|\mathcal{M}_{\beta \alpha}\right| n\right\rangle$.

In summary, there are five contributions to the isotropically averaged oscillator strengths up to second order. Also at this stage it can be verified that the resulting total oscillator strengths are independent of the choice of the origin, which is shown in the Supplementary Material [71]. Note that the individual contributions are still origin dependent. Therefore, a separation into electric and magnetic contributions will also depend on the choice of the origin.

\section{Computational Methodology and Implementation}

The theory presented here for the origin-independent calculation of quadrupole oscillator strengths is applicable in combination with any quantum-chemical method that is capable of providing excited states, either via a time-independent formulation or with response theory (for a review, see, e.g., Ref. [73]). Here, we select TD-DFT which has become an important tool in computational X-ray spectroscopy in the past years $18,23,74,75$. 
We have implemented the calculation of the second-order oscillator strengths into the TDDFT module [76 78] of the Amsterdam density functional (ADF) program package [79,80]. Within TD-DFT, the required electric and magnetic transition moments are calculated as products of the solution vectors $(\boldsymbol{X}+\boldsymbol{Y})$ and $(\boldsymbol{X}-\boldsymbol{Y})$, respectively, with the corresponding matrix elements in the basis of Kohn-Sham molecular orbitals (for details, see, e.g., Refs. 77, 81, 82 ). The required electric-octupole and magnetic-quadrupole integrals are provided by ADF's AORESPONSE module [83, 84].

So far, we have only shown that the theory presented here is origin-independent for the exact eigenfunctions of $\hat{H}_{0}$. However, an additional difficulty arises in approximate calculations. For deriving the equations for the origin-dependence of the magnetic-dipole and magnetic-quadrupole transition moments [Eqs. (54) and (56)], we have converted the occurring electric-dipole and electric-quadrupole transition moments from the velocity to the length representation. However, this is only exact in the case of a complete, infinite basis set. Thus, in calculations using a finite basis set, the magnetic-dipole and magneticquadrupole transition moments do not show the exact origin dependence of Eqs. (54) and $(56)$.

Therefore, we calculate the electric-dipole, electric-quadrupole, and electric-octupole transition moments in the velocity representation for the second-order contributions to the oscillator strengths (see also Refs $[77,85$ for the calculation of transition moments in the velocity representation with TD-DFT). It can be easily verified that this results in second-order oscillator strengths that are origin-independent also in finite basis-set calculations. Note that the electric multipole transition moments in the length and in the velocity representation are only equal in the basis set limit. However, the calculation of higher-order transition moments requires sufficiently large basis sets anyway, so that the values in the length and in the velocity representation are usually in very good agreement.

For the calculation of X-ray absorption spectra in the following, we have employed the 
scheme of Stener et al. 29] to allow only excitations from the relevant core orbital (see also Refs. 30,34 for related schemes). For the $\mathrm{Cl} \mathrm{K}$-edge in $\mathrm{TiCl}_{4}$, only excitations from the $1 s$ orbital of one of the chlorine atoms were considered, while a frozen core was used for the other three chlorine atoms in order to obtain a localized core hole [30]. For the Fe K-edge in vinylferrocene, only excitations from the iron $1 s$ orbital were included. All molecular structures were optimized using the BP86 exchange-correlation functional [86, 87] and ADF's TZP basis set. The TD-DFT calculations were performed using the BP86 functional and the TZ2P basis set and employed a fine numerical integration grid (integration accuracy 8). All calculations were performed with the scalar-relativistic zeroth-order regular approximation (ZORA) 88 91.

\section{Results and Discussion}

To illustrate the origin-independent calculation of quadrupole intensities in X-ray absorption spectroscopy (XAS) using the theory derived above and to verify our implementation, we consider two test cases. As the first example, we use titaniumtetrachloride $\mathrm{TiCl}_{4}$ (see Fig. $2 \mathrm{a}$ for the molecular structure) and calculate the $\mathrm{Cl}$ K-edge XAS spectrum. This example was considered earlier in Refs. 29, 44, 58, For such ligand K-edge spectra, the prepeak transitions are dipole-allowed, and the second-order contribution to the oscillator strength should be small compared to the dipole contribution.

[Figure 2 about here.]

For the lowest-energy $\mathrm{Cl} \mathrm{K}$-edge excitation, the different contributions to the isotropically averaged oscillator strengths are calculated using Eqs (88)-(93), and are listed in Table II for different choices of the origin. In addition, we included the oscillator strengths calculated using the approximation of Ref. [58], i.e., considering only the electric- 
dipole-electric-dipole, electric-quadrupole-electric-quadrupole, and the magnetic-dipolemagnetic-dipole contributions [cf. Eq. (58)] as well as the full second-order oscillator strengths $\left.f_{0 n}^{(0)}+f_{0 n}^{(2)}[\mathrm{cf.} \mathrm{Eq.} 68)\right]$.

The most natural choice for the origin is the chlorine atom from which the $1 s$-electron is excited. In this case, the electric-dipole-electric-dipole contribution $f_{0 n}^{\left(\mu^{2}\right)}$ to the oscillator strength is several orders of magnitude larger than all the second-order contributions, and the approximation of Ref. [58 gives results that are identical to the full secondorder oscillator strengths. In this example, the scheme suggested in Ref. [58] to choose the origin such that the sum of the electric-quadrupole-electric-quadrupole $f_{0 n}^{\left(Q^{2}\right)}$ and the magnetic-dipole-magnetic-dipole contributions $f_{0 n}^{\left(m^{2}\right)}$ is minimized leads to an almost identical choice of the origin. Thus, this scheme is appropriate here.

The situation changes if the origin is not placed at the chlorine atom. To demonstrate this, we moved the origin to the titanium atom. Now, the electric-quadrupole-electricquadrupole $f_{0 n}^{\left(Q^{2}\right)}$ and the magnetic-dipole-magnetic-dipole contributions $f_{0 n}^{\left(m^{2}\right)}$ increase significantly and become several times larger than the dipole oscillator strength $f_{0 n}^{\left(\mu^{2}\right)}$. As a consequence, within the approximation of Ref. [58] the oscillator strength increases by more than a factor of two when shifting the origin from the chlorine to the titanium atom. However, also the magnitudes of electric-dipole-electric-octupole and the electricdipole-magnetic-quadrupole contributions, $f_{0 n}^{(\mu O)}$ and $f_{0 n}^{(\mu \mathcal{M})}$, increase and since these have a negative sign, they exactly cancel the increase of $f_{0 n}^{\left(Q^{2}\right)}$ and $f_{0 n}^{\left(m^{2}\right)}$. Thus, the full secondorder oscillator strength remains unchanged.

In addition, we also shifted the origin away from the molecule by larger amounts. In particular, we used shifts of $10 \AA, 50 \AA$, and $100 \AA$ along the negative $x$-direction. Here, a similar observation can be made. The electric-quadrupole-electric-quadrupole, $f_{0 n}^{\left(Q^{2}\right)}$, and the magnetic-dipole-magnetic-dipole, $f_{0 n}^{\left(m^{2}\right)}$, contributions increase substantially, and for a shift of $100 \AA$, the oscillator strength within the approximation of Ref. [58] is four orders 
of magnitude larger than for the origin at the chlorine atom. On the other hand, when including the electric-dipole-electric-octupole and the electric-dipole-magnetic-quadrupole contributions, the full second-order oscillator strengths are unchanged, even though the individual contributions differ.

\section{[Table 2 about here.]}

As a second example, we consider vinylferrocene, which is a ferrocene molecule bearing a vinyl substituent at one of the cyclopentadienyl rings (see Fig. $2 \mathrm{~b}$ for the molecular structure). Here, we consider the Fe K-edge XAS spectrum and specifically the lowest-energy (prepeak) excitation, which is a $1 s \rightarrow 3 d$ transition. In unsubstituted ferrocene, this prepeak excitation is dipole-forbidden for symmetry reasons, and its oscillator strength is solely due to the second-order contributions. In this case, the electric-quadrupoleelectric-quadrupole and the magnetic-dipole-magnetic-dipole contributions become origin independent (see Section 2.3.5, whereas the remaining second-order contributions $f_{0 n}^{(\mu O)}$ and $f_{0 n}^{(\mu \mathcal{M})}$ vanish. However, in vinylferrocene this symmetry is lost and the lowest-energy transition gains a small dipole oscillator strength (for a detailed discussion, see Ref. [51]).

The oscillator strengths and their contributions calculated for the lowest-energy Fe K-edge excitation using different choices of the origin are shown in Table III. First, the most natural choice for the origin is the iron atom. In this case, the electric-dipole-electric-dipole and the electric-quadrupole-electric-quadrupole contributions to the oscillator strength are comparable in size. The remaining contributions are orders of magnitude smaller. Therefore, the oscillator strength calculated with the approximation of Ref. [58] is identical to the full second-order oscillator strength.

To investigate the dependence on the origin, we shifted the origin far away from the molecule using a shift of $100 \AA$ in the negative $x$-direction, a shift of $100 \AA$ in the negative $z$-direction, and a shift of $50 \AA$ in both the negative $x$-direction and the nega- 
tive $z$-direction. In all three cases, the electric-quadrupole-electric-quadrupole and the magnetic-dipole-magnetic-dipole contributions, $f_{0 n}^{\left(Q^{2}\right)}$ and $f_{0 n}^{\left(m^{2}\right)}$, increase by several orders of magnitude compared to the calculation in which the origin is placed at the iron atom. As a result, the oscillator strengths calculated with the approximation of Ref. 58 also increase by up to five orders of magnitude. However, at the same time the two remaining second-order contributions, i.e., the electric-dipole-electric-octupole contribution $f_{0 n}^{(\mu O)}$ and the electric-dipole-magnetic-quadrupole contribution $f_{0 n}^{(\mu \mathcal{M})}$, assume large negative values and exactly cancel the increase of $f_{0 n}^{\left(Q^{2}\right)}$ and $f_{0 n}^{\left(m^{2}\right)}$ such that the total second-order oscillator strength remains origin independent.

Finally, we used the scheme suggested in Ref. [58] for fixing the origin of the coordinate system, i.e., we chose the origin such that the sum of $f_{0 n}^{\left(Q^{2}\right)}$ and $f_{0 n}^{\left(m^{2}\right)}$ is minimized. In the situation considered here, where the electric-dipole-electric-dipole and the electricquadrupole-electric-quadrupole contributions to the oscillator strengths are of similar size, this scheme moves the origin away from the iron atom. The resulting shift is given in the caption of the last column of Table III. As a consequence, the oscillator strength within the approximation of Ref. [58] decreases by ca. $30 \%$. Again, this decrease is compensated if the remaining second-order contributions are included. Thus, the scheme of Ref. 58 can lead to a spurious decrease of the oscillator strength in some cases. Previously, we found that this problem is even more severe in cases where the electric-dipole-electricdipole contribution to the oscillator strength is significantly smaller than the quadrupole oscillator strength [51. However, if all second-order terms are included consistently the quadrupole oscillator strengths become origin-independent and no special placement of the origin is necessary.

[Table 3 about here.] 


\section{Conclusions}

We have derived origin-independent expressions for calculating XAS intensities beyond the dipole approximation. In particular, we have shown that for a consistent formulation, it is necessary to retain all contributions to the oscillator strengths that are of the same order in the wave vector. This differs from the previous approach [58], in which the multipole expansion was truncated for the transition moments. Here, two additional contributions to the second-order (quadrupole) oscillator strengths arise, which are crossterms depending on products of electric-dipole and electric-octupole transition moments and of electric-dipole and magnetic-quadrupole transition moments, respectively.

Thus, the origin dependence of the sum of electric-quadrupole-electric-quadrupole and magnetic-dipole-magnetic-dipole contributions pointed out earlier [58] is not a fundamental limitation of the use of the multipole expansion. In fact, we could show that to arbitrary order in the wave vector, origin-independent expressions for the oscillator strengths are obtained if all terms of the same order are included consistently. Consequently, within the multipole expansion it should always be possible to derive originindependent expressions for physical observables.

An origin-independent formalism for calculating quadrupole intensities is particularly important for studying ligand and metal K-edge XAS spectra of transition metal complexes. To this end, we have implemented our theory for calculating XAS spectra with TD-DFT, and applied it to two simple test cases. Here, we want to stress that our results do not invalidate any previous results obtained with the formalism of Ref. [58. On the contrary, our test calculations showed that the two additional contributions are negligible as long as the origin of the coordinate system is placed at the atom where the core excitation occurs. However, with our origin-independent theory, it is no longer necessary to make sure that the origin is chosen appropriately. This is particularly important for cases where the 
quadrupole intensity is larger than or comparable to the dipole contribution, where the scheme proposed in Ref. [58 might place the origin far away from the relevant core orbital. Moreover, it makes it possible to treat excitations from core orbitals that are delocalized over several atoms (e.g., for calculating ligand K-edge spectra or metal K-edge spectra in polynuclear transition metal complexes) without the need to perform a transformation to localized core orbitals.

Of course, the theory presented here is not limited to TD-DFT, but can be employed for the calculation of quadrupole intensities in combination with any quantum-chemical method capable of providing the required transition moments. Moreover, it is not restricted to XAS spectroscopy, but is also applicable for calculating XES intensities, for instance using the approach of Ref. [59]. Finally, we note that it becomes necessary to go beyond the dipole approximation, not only for short wavelengths, such as those employed in hard X-ray spectroscopy, but also for extended molecular systems. For describing the optical response of an extended nanostructure in the visible spectrum, it becomes necessary to go beyond the dipole approximation as well. Thus, the origin-independent formalism derived here will also be essential for predicting optical properties of nanostructured materials, such as, for instance, metamaterials 92 .

\section{Acknowledgments}

We thank Prof. Wim Klopper (KIT) for inspiring this work and for helpful discussions. Funding from the DFG-Center for Functional Nanostructures is gratefully acknowledged. 


\section{Appendix}

\section{A Length and velocity representation}

To show how the electric-multipole moments in the velocity representation can be converted to those in the conventional length representation, we use the following commutators of (products of) the Cartesian components of the position operator with the molecular Hamiltonian given in Eq. (7),

$$
\begin{aligned}
{\left[r_{i, \alpha}, \hat{H}_{0}\right] } & =\frac{\mathrm{i} \hbar}{m} \hat{p}_{i, \alpha}, \\
{\left[r_{i, \alpha} r_{i, \beta}, \hat{H}_{0}\right] } & =\frac{\mathrm{i} \hbar}{m}\left(\hat{p}_{i, \alpha} r_{i, \beta}+r_{i, \alpha} \hat{p}_{i, \beta}\right), \\
{\left[r_{i, \alpha} r_{i, \beta} r_{i, \gamma}, \hat{H}_{0}\right] } & =\frac{\mathrm{i} \hbar}{m}\left(\hat{p}_{i, \alpha} r_{i, \beta} r_{i, \gamma}+r_{i, \alpha} \hat{p}_{i, \beta} r_{i, \gamma}+r_{i, \alpha} r_{i, \beta} \hat{p}_{i, \gamma}\right) .
\end{aligned}
$$

Next, we employ that the matrix elements of the commutator of an operator $\hat{A}$ and $\hat{H}_{0}$ are given by

$$
\left\langle 0\left|\left[\hat{A}, \hat{H}_{0}\right]\right| n\right\rangle=\left\langle 0\left|\hat{A} \hat{H}_{0}-\hat{H}_{0} \hat{A}\right| n\right\rangle=E_{n}\langle 0|\hat{A}| n\rangle-E_{0}\langle 0|\hat{A}| n\rangle=E_{0 n}\langle 0|\hat{A}| n\rangle
$$

Here, it is important to point out that this relation is only valid for the exact eigenfunctions of $\hat{H}_{0}$ and that it only holds approximately for approximate wavefunctions.

Now, we can use these results to obtain

$$
\left\langle 0\left|\hat{p}_{i, \alpha}\right| n\right\rangle=\frac{m}{\mathrm{i} \hbar}\left\langle 0\left|\left[r_{i, \alpha}, \hat{H}_{0}\right]\right| n\right\rangle=-\mathrm{i} E_{0 n} \frac{m}{\hbar}\left\langle 0\left|r_{i, \alpha}\right| n\right\rangle
$$

and get for the electric-dipole transition moments

$$
\left\langle 0\left|\hat{\mu}_{\alpha}^{p}\right| n\right\rangle=\sum_{i} \frac{e}{m}\left\langle 0\left|\hat{p}_{i, \alpha}\right| n\right\rangle=-\mathrm{i} \frac{E_{0 n}}{\hbar} e \sum_{i}\left\langle 0\left|r_{i, \alpha}\right| n\right\rangle=-\mathrm{i} \frac{E_{0 n}}{\hbar}\left\langle 0\left|\hat{\mu}_{\alpha}\right| n\right\rangle .
$$

Similarly, we find for the electric-quadrupole transition moments

$$
\left\langle 0\left|\hat{Q}_{\alpha \beta}^{p}\right| n\right\rangle=-\mathrm{i} \frac{E_{0 n}}{\hbar}\left\langle 0\left|\hat{Q}_{\alpha \beta}\right| n\right\rangle
$$


and for the electric-octupole transition moments

$$
\left\langle 0\left|\hat{O}_{\alpha \beta \gamma}^{p}\right| n\right\rangle=-\mathrm{i} \frac{E_{0 n}}{\hbar}\left\langle 0\left|\hat{O}_{\alpha \beta \gamma}\right| n\right\rangle
$$

\section{B Antisymmetric second-order term}

First, the matrix elements in the antisymmetric term in Eq. (40), can be split as

$$
\begin{aligned}
& 2\left(\boldsymbol{k} \cdot \boldsymbol{r}_{i}\right)\left(\boldsymbol{k} \cdot \boldsymbol{r}_{i}\right)\left(\hat{\boldsymbol{p}}_{i} \cdot \mathcal{E}\right)-\left(\boldsymbol{k} \cdot \boldsymbol{r}_{i}\right)\left(\boldsymbol{k} \cdot \hat{\boldsymbol{p}}_{i}\right)\left(\boldsymbol{r}_{i} \cdot \mathcal{E}\right)-\left(\boldsymbol{k} \cdot \hat{\boldsymbol{p}}_{i}\right)\left(\boldsymbol{k} \cdot \boldsymbol{r}_{i}\right)\left(\boldsymbol{r}_{i} \cdot \mathcal{E}\right) \\
= & \left(\boldsymbol{k} \cdot \boldsymbol{r}_{i}\right)\left(\boldsymbol{k} \cdot \boldsymbol{r}_{i}\right)\left(\hat{\boldsymbol{p}}_{i} \cdot \mathcal{E}\right)-\left(\boldsymbol{k} \cdot \boldsymbol{r}_{i}\right)\left(\boldsymbol{k} \cdot \hat{\boldsymbol{p}}_{i}\right)\left(\boldsymbol{r}_{i} \cdot \mathcal{E}\right) \\
& +\left(\boldsymbol{k} \cdot \boldsymbol{r}_{i}\right)\left(\boldsymbol{k} \cdot \boldsymbol{r}_{i}\right)\left(\hat{\boldsymbol{p}}_{i} \cdot \mathcal{E}\right)-\left(\boldsymbol{k} \cdot \hat{\boldsymbol{p}}_{i}\right)\left(\boldsymbol{k} \cdot \boldsymbol{r}_{i}\right)\left(\boldsymbol{r}_{i} \cdot \mathcal{E}\right) .
\end{aligned}
$$

For both terms, we can employ that both $\left(\boldsymbol{k} \cdot \boldsymbol{r}_{i}\right)$ and $\left(\hat{\boldsymbol{p}}_{i} \cdot \mathcal{E}\right)$ commute because $\boldsymbol{k}$ and $\mathcal{E}$ are orthogonal, and subsequently use the vector identity of Eq. (31). In the same fashion as for the antisymmetric first-order contribution, we then obtain for the first term,

$$
\begin{aligned}
&\left(\boldsymbol{k} \cdot \boldsymbol{r}_{i}\right) {\left[\left(\boldsymbol{k} \cdot \boldsymbol{r}_{i}\right)\left(\hat{\boldsymbol{p}}_{i} \cdot \mathcal{E}\right)-\left(\boldsymbol{k} \cdot \hat{\boldsymbol{p}}_{i}\right)\left(\boldsymbol{r}_{i} \cdot \mathcal{E}\right)\right] } \\
&=\left(\boldsymbol{k} \cdot \boldsymbol{r}_{i}\right)(\boldsymbol{k} \times \mathcal{E}) \cdot\left(\boldsymbol{r}_{i} \times \hat{\boldsymbol{p}}_{i}\right)=(\boldsymbol{k} \times \mathcal{E}) \cdot\left(\boldsymbol{k} \cdot \boldsymbol{r}_{i}\right)\left(\boldsymbol{r}_{i} \times \hat{\boldsymbol{p}}_{i}\right),
\end{aligned}
$$

and for the second term, we get,

$$
\begin{aligned}
\left(\boldsymbol{k} \cdot \boldsymbol{r}_{i}\right) & \left(\boldsymbol{k} \cdot \boldsymbol{r}_{i}\right)\left(\hat{\boldsymbol{p}}_{i} \cdot \mathcal{E}\right)-\left(\boldsymbol{k} \cdot \hat{\boldsymbol{p}}_{i}\right)\left(\boldsymbol{k} \cdot \boldsymbol{r}_{i}\right)\left(\boldsymbol{r}_{i} \cdot \mathcal{E}\right) \\
= & \left(\boldsymbol{k} \cdot \boldsymbol{r}_{i}\right)\left(\hat{\boldsymbol{p}}_{i} \cdot \mathcal{E}\right)\left(\boldsymbol{k} \cdot \boldsymbol{r}_{i}\right)-\left(\boldsymbol{k} \cdot \hat{\boldsymbol{p}}_{i}\right)\left(\boldsymbol{r}_{i} \cdot \mathcal{E}\right)\left(\boldsymbol{k} \cdot \boldsymbol{r}_{i}\right) \\
= & {\left[\left(\boldsymbol{k} \cdot \boldsymbol{r}_{i}\right)\left(\hat{\boldsymbol{p}}_{i} \cdot \mathcal{E}\right)-\left(\boldsymbol{k} \cdot \hat{\boldsymbol{p}}_{i}\right)\left(\boldsymbol{r}_{i} \cdot \mathcal{E}\right)\right]\left(\boldsymbol{k} \cdot \boldsymbol{r}_{i}\right) } \\
& =(\boldsymbol{k} \times \mathcal{E}) \cdot\left(\boldsymbol{r}_{i} \times \hat{\boldsymbol{p}}_{i}\right)\left(\boldsymbol{k} \cdot \boldsymbol{r}_{i}\right) .
\end{aligned}
$$

Altogether, we arrive at

$$
\begin{aligned}
& 2\left(\boldsymbol{k} \cdot \boldsymbol{r}_{i}\right)\left(\boldsymbol{k} \cdot \boldsymbol{r}_{i}\right)\left(\hat{\boldsymbol{p}}_{i} \cdot \mathcal{E}\right)-\left(\boldsymbol{k} \cdot \boldsymbol{r}_{i}\right)\left(\boldsymbol{k} \cdot \hat{\boldsymbol{p}}_{i}\right)\left(\boldsymbol{r}_{i} \cdot \mathcal{E}\right)-\left(\boldsymbol{k} \cdot \hat{\boldsymbol{p}}_{i}\right)\left(\boldsymbol{k} \cdot \boldsymbol{r}_{i}\right)\left(\boldsymbol{r}_{i} \cdot \mathcal{E}\right) \\
= & (\boldsymbol{k} \times \mathcal{E}) \cdot\left[\left(\boldsymbol{k} \cdot \boldsymbol{r}_{i}\right) \cdot\left(\boldsymbol{r}_{i} \times \hat{\boldsymbol{p}}_{i}\right)+\left(\boldsymbol{r}_{i} \times \hat{\boldsymbol{p}}_{i}\right)\left(\boldsymbol{k} \cdot \boldsymbol{r}_{i}\right)\right] .
\end{aligned}
$$




\section{Origin independence in arbitrary order}

In Section 2.4.1, we showed explicitly that the second-order oscillator strengths are independent of the choice of the origin. Here, we prove that this still holds for an arbitrary order. From the definition of the full transition moments [Eq. (14)], we find for its change upon shifting the origin from $\boldsymbol{O}$ to $\boldsymbol{O}+\boldsymbol{a}$,

$$
T(\boldsymbol{O}+\boldsymbol{a})=\exp (\mathrm{i} \boldsymbol{k} \cdot \boldsymbol{a}) T(\boldsymbol{O})=\left(\sum_{n=0}^{\infty} \frac{\mathrm{i}^{n}}{n !}(\boldsymbol{k} \cdot \boldsymbol{a})^{n}\right)\left(\sum_{n=0}^{\infty} T^{(n)}(\boldsymbol{O})\right),
$$

and can identify the terms that are of order $m$ in the wave vector,

$$
T^{(m)}(\boldsymbol{O}+\boldsymbol{a})=\sum_{n=0}^{m} \frac{\mathrm{i}^{n}}{n !}(\boldsymbol{k} \cdot \boldsymbol{a})^{n} T^{(m-n)}(\boldsymbol{O})
$$

For the oscillator strengths, the terms that are of order $m$ in the wave vector are,

$$
f^{(m)}(\boldsymbol{O})=\frac{2 m_{e}}{e^{2} E_{0 n}} \sum_{n=0}^{m} T^{(n)}(\boldsymbol{O})\left[T^{(m-n)}(\boldsymbol{O})\right]^{*},
$$

and when the origin of the coordinate system is shifted, this becomes

$$
\begin{aligned}
f^{(m)}(\boldsymbol{O}+\boldsymbol{a}) & =\frac{2 m_{e}}{e^{2} E_{0 n}} \sum_{n=0}^{m} T^{(n)}(\boldsymbol{O}+\boldsymbol{a})\left[T^{(m-n)}(\boldsymbol{O}+\boldsymbol{a})\right]^{*} \\
& =\frac{2 m_{e}}{e^{2} E_{0 n}} \sum_{n=0}^{m}\left[\sum_{p=0}^{n} \frac{\mathrm{i}^{p}}{p !}(\boldsymbol{k} \cdot \boldsymbol{a})^{p} T^{(n-p)}(\boldsymbol{O})\right]\left[\sum_{q=0}^{m-n} \frac{(-\mathrm{i})^{q}}{q !}(\boldsymbol{k} \cdot \boldsymbol{a})^{q}\left[T^{(m-n-q)}(\boldsymbol{O})\right]^{*}\right] \\
& =\frac{2 m_{e}}{e^{2} E_{0 n}} \sum_{n=0}^{m} \sum_{p=0}^{n} \sum_{q=0}^{m-n}(-1)^{q} \frac{\mathrm{i}^{p+q}}{p ! q !}(\boldsymbol{k} \cdot \boldsymbol{a})^{p+q} T^{(n-p)}(\boldsymbol{O})\left[T^{(m-n-q)}(\boldsymbol{O})\right]^{*} .
\end{aligned}
$$

Now we eliminate $p$ and $q$ by introducing the new indices $r=p+q$ and $s=n-p$ to arrive at

$$
f^{(m)}(\boldsymbol{O}+\boldsymbol{a})=\frac{2 m_{e}}{e^{2} E_{0 n}} \sum_{n=0}^{m} \sum_{s=0}^{n} \sum_{r=n-s}^{m-s} \frac{(-1)^{r-n+s} \mathrm{i}^{r}}{(n-s) !(r-n+s) !}(\boldsymbol{k} \cdot \boldsymbol{a})^{r} T^{(s)}(\boldsymbol{O})\left[T^{(m-r-s)}(\boldsymbol{O})\right]^{*}
$$


The three sums can be rewritten and put in a different order, which leads to

$$
\begin{aligned}
f^{(m)}(\boldsymbol{O}+\boldsymbol{a})= & \frac{2 m_{e}}{e^{2} E_{0 n}} \sum_{r=0}^{m}(\boldsymbol{k} \cdot \boldsymbol{a})^{r} \sum_{s=0}^{m-r} T^{(s)}(\boldsymbol{O})\left[T^{(m-r-s)}(\boldsymbol{O})\right]^{*} \sum_{n=s}^{s+r} \frac{(-1)^{r-n+s} \mathrm{i}^{r}}{(n-s) !(r-n+s) !} \\
= & f^{(m)}(\boldsymbol{O}) \\
& +\frac{2 m_{e}}{e^{2} E_{0 n}} \sum_{r=1}^{m}(\boldsymbol{k} \cdot \boldsymbol{a})^{r} \sum_{s=0}^{m-r} T^{(s)}(\boldsymbol{O})\left[T^{(m-r-s)}(\boldsymbol{O})\right]^{*} \sum_{t=0}^{r} \frac{(-1)^{r-t} \mathrm{i}^{r}}{t !(r-t) !}
\end{aligned}
$$

That the summation here is equivalent to the one in Eq. C-5 can be seen easily by considering the six inequalities corresponding to the sums in the two cases and showing that these are equivalent. In the second line above, we have taken the term $m=0$ out for the first sum and introduced the new index $t=n-s$.

Finally, we can use the binomial theorem to realize that,

$$
0=(\mathrm{i}-\mathrm{i})^{r}=\sum_{t=0}^{r} \frac{r !}{t !(r-t) !} \mathrm{i}^{t}(-\mathrm{i})^{r-t}=r ! \sum_{t=0}^{r} \frac{(-1)^{r-t} \mathrm{i}^{r}}{t !(r-t) !},
$$

that is, the last term in the above equation is zero and, thus, we have shown that in any order $k$, the oscillator strengths are origin-independent.

\section{References}

[1] D. C. Koningsberger and R. Prins, editors, X-Ray Absorption: Principles, Applications, Techniques of EXAFS, SEXAFS and XANES, Wiley, New York, 1988.

[2] J. Stöhr, NEXAFS Spectroscopy, Springer, Berlin, 1992.

[3] J. Singh, C. Lamberti, and J. A. v. Bokhoven, Chem. Soc. Rev. 39, 4754 (2010).

[4] Q. X. Guo et al., Appl. Phys. Lett. 98, 181901 (2011).

[5] R. Schuber, P. R. Ganz, F. Wilhelm, A. Rogalev, and D. M. Schaadt, Phys. Rev. B 84, 155206 (2011). 
[6] S. Gross and M. Bauer, Adv. Funct. Mater. 20, 4026-4047 (2010).

[7] M. Bauer and C. Gastl, Phys. Chem. Chem. Phys. 12, 5575 (2010).

[8] E. I. Solomon, R. K. Szilagyi, S. DeBeer George, and L. Basumallick, Chem. Rev. 104, 419 (2004).

[9] C. J. Pollock and S. DeBeer, J. Am. Chem. Soc. 133, 5594 (2011).

[10] J. Singh, R. C. Nelson, B. C. Vicente, S. L. Scott, and J. A. v. Bokhoven, Phys. Chem. Chem. Phys. 12, 5668 (2010).

[11] E. Kleymenov, J. Sa, J. Abu-Dahrieh, D. Rooney, J. A. v. Bokhoven, E. Troussard, J. Szlachetko, O. V. Safonova, and M. Nachtegaal, Catal. Sci. Technol. 2, 373 (2012).

[12] K. Ray, T. Petrenko, K. Wieghardt, and F. Neese, Dalton Trans. 2007, 1552 (2007).

[13] J. F. Berry, S. D. George, and F. Neese, Phys. Chem. Chem. Phys. 10, 4361 (2008).

[14] P. Chandrasekaran, S. C. E. Stieber, T. J. Collins, J. Lawrence Que, F. Neese, and S. DeBeer, Dalton Trans. 40, 11070 (2011).

[15] N. Sun, L. V. Liu, A. Dey, G. Villar-Acevedo, J. A. Kovacs, M. Y. Darensbourg, K. O. Hodgson, B. Hedman, and E. I. Solomon, Inorg. Chem. 50, 427 (2011).

[16] K. M. Lancaster, M. Roemelt, P. Ettenhuber, Y. Hu, M. W. Ribbe, F. Neese, U. Bergmann, and S. DeBeer, Science 334, 974 (2011).

[17] M. A. Beckwith, M. Roemelt, M.-N. Collomb, C. DuBoc, T.-C. Weng, U. Bergmann, P. Glatzel, F. Neese, and S. DeBeer, Inorg. Chem. 50, 8397 (2011).

[18] M. Roemelt, M. A. Beckwith, C. Duboc, M.-N. Collomb, F. Neese, and S. DeBeer, Inorg. Chem. 51, 680 (2012). 
[19] V. Carravetta, H. Ågren, and V. Barone, Computational X-Ray Spectroscopy, in Computational Strategies for Spectroscopy: from Small Molecules to Nano Systems, pages 137-205, Wiley, Hoboken, New Jersey, 2011.

[20] J. J. Rehr and R. C. Albers, Rev. Mod. Phys. 72, 621 (2000).

[21] J. J. Rehr and A. L. Ankudinov, Coord. Chem. Rev. 249, 131 (2005).

[22] F. Gel'mukhanov and H. Ågren, Phys. Rep. 312, 87 (1999).

[23] N. A. Besley and F. A. Asmuruf, Phys. Chem. Chem. Phys. 12, 12024 (2010).

[24] H. Ågren, V. Carravetta, O. Vahtras, and L. G. M. Pettersson, Chem. Phys. Lett. 222, 75 (1994).

[25] H. Ågren, V. Carravetta, O. Vahtras, and L. G. M. Pettersson, Theor. Chem. Acc. 97, $14(1997)$.

[26] U. Ekström, P. Norman, and V. Carravetta, Phys. Rev. A 73, 022501 (2006).

[27] L. Triguero, L. G. M. Pettersson, and H. Ågren, Phys. Rev. B 58, 8097 (1998).

[28] M. Leetmaa, M. P. Ljungberg, A. Lyubartsev, A. Nilsson, and L. G. M. Pettersson, J. Electron Spectrosc. Relat. Phenom. 177, 135 (2010).

[29] M. Stener, G. Fronzoni, and M. de Simone, Chem. Phys. Lett. 373, 115 (2003).

[30] K. Ray, S. DeBeer George, E. I. Solomon, K. Wieghardt, and F. Neese, Chem.-Eur. J. 13, 2783-2797 (2007).

[31] T. Tsuchimochi, M. Kobayashi, A. Nakata, Y. Imamura, and H. Nakai, J. Comput. Chem. 29, 2311-2316 (2008).

[32] N. Schmidt, R. Fink, and W. Hieringer, J. Chem. Phys. 133, 054703 (2010).

[33] A. Kovyrshin and J. Neugebauer, J. Chem. Phys. 133, 174114 (2010). 
[34] W. Liang, S. A. Fischer, M. J. Frisch, and X. Li, J. Chem. Theory Comput. 7, 3540 (2011).

[35] U. Ekström, P. Norman, V. Carravetta, and H. Ågren, Phys. Rev. Lett. 97, 143001 (2006).

[36] U. Ekström and P. Norman, Phys. Rev. A 74, 042722 (2006).

[37] K. Lopata, B. E. Van Kuiken, M. Khalil, and N. Govind, J. Chem. Theory Comput. 8, $3284(2012)$.

[38] S. Coriani, O. Christiansen, T. Fransson, and P. Norman, Phys. Rev. A 85, 022507 (2012).

[39] S. Coriani, T. Fransson, O. Christiansen, and P. Norman, J. Chem. Theory Comput. 8, $1616(2012)$.

[40] S. E. Shadle, B. Hedman, K. O. Hodgson, and E. I. Solomon, J. Am. Chem. Soc. 117, 2259 (1995).

[41] T. Glaser, B. Hedman, K. O. Hodgson, and E. I. Solomon, Acc. Chem. Res. 33, 859 (2000).

[42] L.-Å. Näslund, M. Cavalleri, H. Ogasawara, A. Nilsson, L. G. M. Pettersson, P. Wernet, D. C. Edwards, M. Sandström, and S. Myneni, J. Phys. Chem. A 107, 6869 (2003).

[43] E. I. Solomon, B. Hedman, K. O. Hodgson, A. Dey, and R. K. Szilagyi, Coord. Chem. Rev. 249, 97 (2005).

[44] S. DeBeer George, P. Brant, and E. I. Solomon, J. Am. Chem. Soc. 127, 667 (2005).

[45] S. DeBeer George and F. Neese, Inorg. Chem. 49, 1849 (2010). 
[46] A. Dey, Y. Peng, W. E. Broderick, B. Hedman, K. O. Hodgson, J. B. Broderick, and E. I. Solomon, J. Am. Chem. Soc. 133, 18656 (2011).

[47] T. E. Westre, P. Kennepohl, J. G. DeWitt, B. Hedman, K. O. Hodgson, and E. I. Solomon, J. Am. Chem. Soc. 119, 6297 (1997).

[48] S. DeBeer George, T. Petrenko, and F. Neese, J. Phys. Chem. A 112, 12936 (2008).

[49] R. K. Hocking, S. D. George, Z. Gross, F. A. Walker, K. O. Hodgson, B. Hedman, and E. I. Solomon, Inorg. Chem. 48, 1678 (2009).

[50] C. C. Scarborough, S. Sproules, T. Weyhermüller, S. DeBeer, and K. Wieghardt, Inorg. Chem. 50, 12446 (2011).

[51] A. J. Atkins, Ch. R. Jacob, and M. Bauer, Chem.-Eur. J. 18, 7021-7025 (2012).

[52] K. Hämäläinen, D. P. Siddons, J. B. Hastings, and L. E. Berman, Phys. Rev. Lett. 67, 2850 (1991).

[53] F. M. F. de Groot, M. H. Krisch, and J. Vogel, Phys. Rev. B 66, 195112 (2002).

[54] P. Glatzel and U. Bergmann, Coord. Chem. Rev. 249, 65 (2005).

[55] G. R. Shulman, Y. Yafet, P. Eisenberger, and W. E. Blumberg, Proc. Natl. Acad. Sci. U. S. A. 73, 1384 (1976).

[56] G. Dräger, R. Frahm, G. Materlik, and O. Brümmer, Phys. Status Solidi B 146, 287-294 (1988).

[57] T. Yamamoto, X-Ray Spectrom. 37, 572-584 (2008).

[58] S. DeBeer George, T. Petrenko, and F. Neese, Inorg. Chim. Acta 361, 965 (2008).

[59] N. Lee, T. Petrenko, U. Bergmann, F. Neese, and S. DeBeer, J. Am. Chem. Soc. 132, $9715(2010)$. 
[60] M. Reiher and A. Wolf, Relativistic Quantum Chemistry: The Fundamental Theory of Molecular Science, Wiley-VCH, Weinheim, 2009.

[61] J. D. Jackson, Classical Electrodynamics, Wiley, New York, 3rd edition, 1998.

[62] C. Cohen-Tannoudji, B. Diu, and F. Laloe, Quantum Mechanics, Vol. 2, Wiley, New York, 1978.

[63] G. C. Schatz and M. A. Ratner, Quantum Mechanics in Chemistry, Dover Publications, Mineola, N.Y., 2002.

[64] F. Schwabl, Quantenmechanik: Eine Einführung, Springer, Berlin/Heidelberg, 6th edition, 2002.

[65] P. W. Atkins and R. S. Friedman, Molecular Quantum Mechanics, Oxford University Press, Oxford, 5th edition, 2010.

[66] J. Lehtola, M. Hakala, A. Sakko, and K. Hämäläinen, J. Comput. Chem. 33, $1572-1585$ (2012).

[67] L. D. Barron, Molecular Light Scattering and Optical Activity, Cambridge University Press, Cambridge, 2nd edition, 2004.

[68] E. B. Graham and R. E. Raab, Proc. Roy. Soc. Ser. A 430, 593 (1990).

[69] R. McWeeny, Spins in Chemistry, Dover Publications, Mineola, N.Y., 2004.

[70] Ch. R. Jacob and M. Reiher, Int. J. Quantum Chem. 112, 3661 (2012).

[71] See supplementary material at http://dx.doi.org/10.1063/1.4766359 for derivation of the equations for the origin dependence of the multipole transition moments and of the second-order oscillator strengths.

[72] E. B. Graham and R. E. Raab, Proc. Roy. Soc. Ser. A 456, 1193 (2000). 
[73] A. S. P. Gomes and Ch. R. Jacob, Annu. Rep. Prog. Chem. C 108, 222 (2012).

[74] N. A. Besley, M. J. G. Peach, and D. J. Tozer, Phys. Chem. Chem. Phys. 11, 10350 (2009).

[75] F. Neese, Coord. Chem. Rev. 253, 526 (2009).

[76] S. J. A. van Gisbergen, J. G. Snijders, and E. J. Baerends, Comput. Phys. Commun. 118, 119 (1999).

[77] J. Autschbach and T. Ziegler, J. Chem. Phys. 116, 891 (2002).

[78] J. Autschbach, T. Ziegler, S. J. A. van Gisbergen, and E. J. Baerends, J. Chem. Phys. 116, 6930 (2002).

[79] Theoretical Chemistry, Vrije Universiteit Amsterdam, ADF, Amsterdam density functional program, URL: http://www.scm.com.

[80] G. te Velde, F. M. Bickelhaupt, E. J. Baerends, C. Fonseca Guerra, S. J. A. van Gisbergen, J. G. Snijders, and T. Ziegler, J. Comput. Chem. 22, 931 (2001).

[81] M. E. Casida, Time-Dependent Density Functional Response Theory for Molecules, in Recent Advances in Density-Functional Methods, edited by D. P. Chong, pages 155-192, World Scientific, Singapore, 1995.

[82] F. Furche, J. Chem. Phys. 114, 5982 (2001).

[83] M. Krykunov, A. Banerjee, T. Ziegler, and J. Autschbach, J. Chem. Phys. 122, 074105 (2005).

[84] M. Krykunov and J. Autschbach, J. Chem. Phys. 126, 024101 (2007).

[85] S. Grimme, F. Furche, and R. Ahlrichs, Chem. Phys. Lett. 361, 321 (2002).

[86] A. D. Becke, Phys. Rev. A 38, 3098 (1988). 
[87] J. P. Perdew, Phys. Rev. B 33, 8822 (1986).

[88] E. van Lenthe, E. J. Baerends, and J. G. Snijders, J. Chem. Phys. 99, 4597 (1993).

[89] E. van Lenthe, E. J. Baerends, and J. G. Snijders, J. Chem. Phys. 101, 9783 (1994).

[90] E. van Lenthe, J. G. Snijders, and E. J. Baerends, J. Chem. Phys. 105, 6505 (1996).

[91] E. van Lenthe, A. Ehlers, and E.-J. Baerends, J. Chem. Phys. 110, 8943 (1999).

[92] R. Merlin, Proc. Natl. Acad. Sci. U. S. A. 106, 1693 (2009). 


\section{List of Figures}

1 Schematic illustration of the different terms arising from the squared absolute value in Eq. (57). The entries in the table indicate the order of the different terms in the wave vector $\boldsymbol{k}$. We retain all terms up to second order, as indicated by the red line. . . . . . . . . . . . . . . . 45

2 Molecular structures of the model systems considered for the calculation of X-ray absorption spectra. (a) Titaniumtetrachloride $\left(\mathrm{TiCl}_{4}\right)$ and (b) Vinylferrocene. The orientation of the molecules within the coordinate system is also indicated. . . . . . . . . . . . . . 46 
Figure 1: Schematic illustration of the different terms arising from the squared absolute value in Eq. (57). The entries in the table indicate the order of the different terms in the wave vector $\boldsymbol{k}$. We retain all terms up to second order, as indicated by the red line.

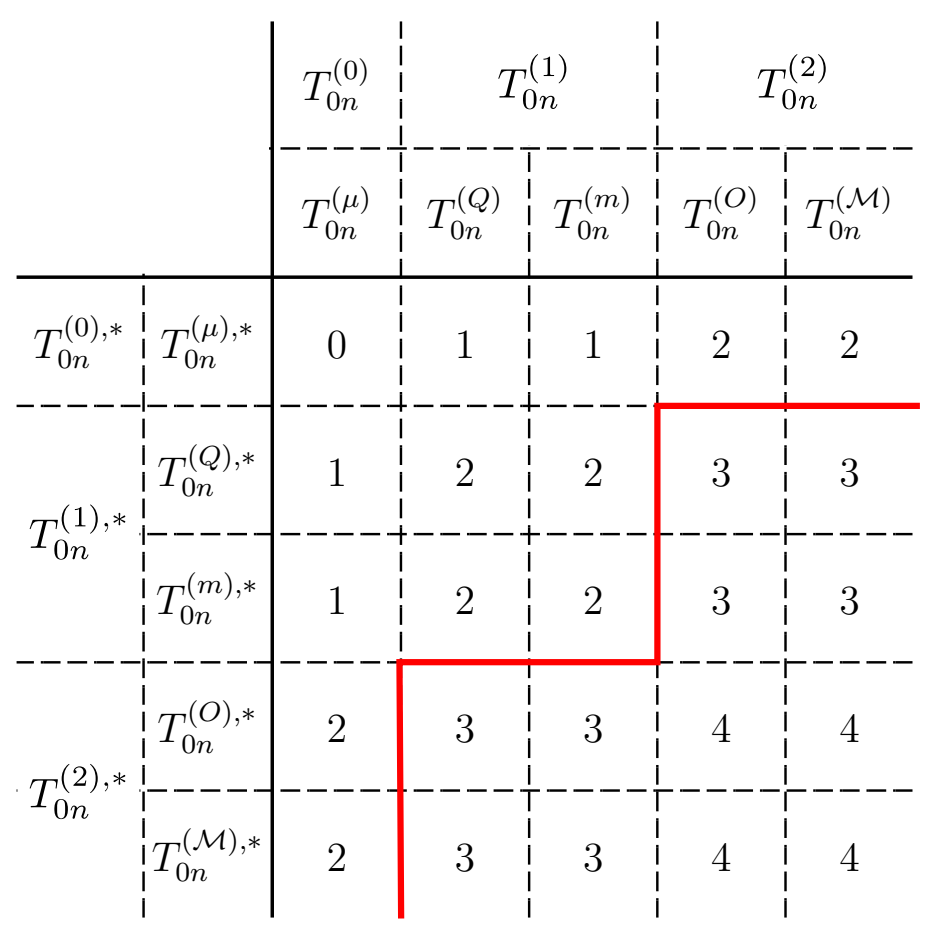


Figure 2: Molecular structures of the model systems considered for the calculation of X-ray absorption spectra. (a) Titaniumtetrachloride $\left(\mathrm{TiCl}_{4}\right)$ and (b) Vinylferrocene. The orientation of the molecules within the coordinate system is also indicated.

(a)

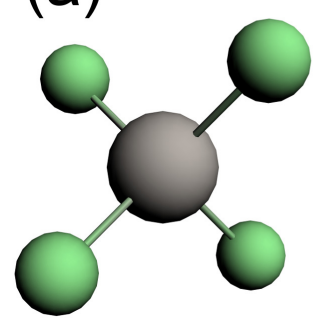

(b)
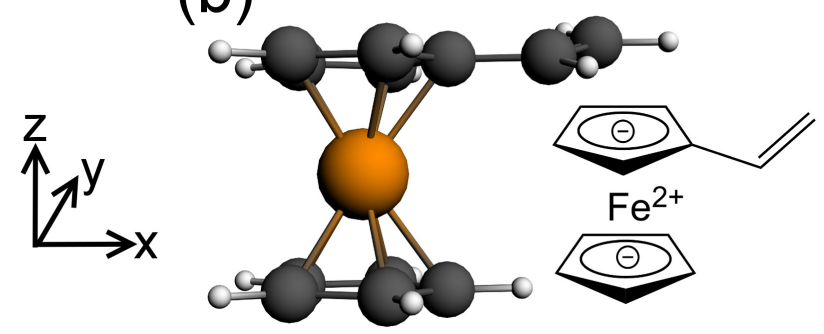


\section{List of Tables}

I Overview of the different contributions appearing in the multipole expansion of the transition moments of Eq. (14) up to second order. . . . . . . . 48

II X-ray absorption oscillator strength for $\mathrm{TiCl}_{4}$, calculated for the lowestenergy transition at the $\mathrm{Cl}$ K-edge (excitation energy $2755.6 \mathrm{eV}$ ). The total isotropically averaged oscillator strength and its different contributions are given for different positions of the origin of the coordinate system. . . . . . 49

III X-ray absorption oscillator strength for Vinylferrocene, calculated for the lowest-energy transition at the Fe K-edge (excitation energy $7051.3 \mathrm{eV}$ ). The total isotropically averaged oscillator strength and its different contributions are given for different positions of the origin of the coordinate

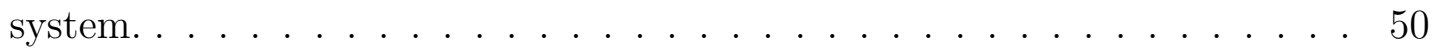


Table I: Overview of the different contributions appearing in the multipole expansion of the transition moments of Eq. (14) up to second order.

\begin{tabular}{ccc}
\hline \hline order & electric & magnetic \\
\hline 0 & $T_{0 n}^{(\mu)}=-\mathrm{i} \frac{E_{0 n}}{\hbar} \sum_{\alpha} \mathcal{E}_{\alpha}\left\langle 0\left|\hat{\mu}_{\alpha}\right| n\right\rangle$ & - \\
1 & $T_{0 n}^{(Q)}=\frac{E_{0 n}}{2 \hbar} \sum_{\alpha \beta} k_{\alpha} \mathcal{E}_{\beta}\left\langle 0\left|\hat{Q}_{\alpha \beta}\right| n\right\rangle$ & $T_{0 n}^{(m)}=\mathrm{i} c \sum_{\alpha}(\boldsymbol{k} \times \mathcal{E})_{\alpha}\left\langle 0\left|\hat{m}_{\alpha}\right| n\right\rangle$ \\
2 & $T_{0 n}^{(O)}=\mathrm{i} \frac{E_{0 n}}{6 \hbar} \sum_{\alpha \beta \gamma} k_{\alpha} k_{\beta} \mathcal{E}_{\gamma}\left\langle 0\left|\hat{O}_{\alpha \beta \gamma}\right| n\right\rangle$ & $T_{0 n}^{(\mathcal{M})}=-\frac{c}{2} \sum_{\alpha \beta}(\boldsymbol{k} \times \mathcal{E})_{\alpha} k_{\beta}\left\langle 0\left|\hat{\mathcal{M}}_{\alpha \beta}\right| n\right\rangle$ \\
\hline \hline
\end{tabular}


Table II: X-ray absorption oscillator strength for $\mathrm{TiCl}_{4}$, calculated for the lowest-energy transition at the $\mathrm{Cl} \mathrm{K}$-edge (excitation energy $2755.6 \mathrm{eV}$ ). The total isotropically averaged oscillator strength and its different contributions are given for different positions of the origin of the coordinate system.

\begin{tabular}{cccccc}
\hline \hline origin & & $\boldsymbol{O}$ & $\boldsymbol{O}+\boldsymbol{a}$ & $\boldsymbol{O}+\boldsymbol{a}$ & $\boldsymbol{O}+\boldsymbol{a}$ \\
& $\mathrm{Cl}$ atom & Ti atom & $a_{x}=-10 \AA$ & $a_{x}=-50 \AA$ & $a_{x}=-100 \AA$ \\
\hline$\left\langle f_{0 n}^{\left(\mu^{2}\right)}\right\rangle_{\text {iso }}$ & $4.57 \cdot 10^{-4}$ & $4.57 \cdot 10^{-4}$ & $4.58 \cdot 10^{-4}$ & $4.57 \cdot 10^{-4}$ & $4.58 \cdot 10^{-4}$ \\
$\left\langle f_{0 n}^{\left(Q^{2}\right)}\right\rangle_{\text {iso }}$ & $6.04 \cdot 10^{-10}$ & $7.24 \cdot 10^{-4}$ & $1.09 \cdot 10^{-2}$ & $3.65 \cdot 10^{-1}$ & 1.35 \\
$\left\langle f_{0 n}^{\left(m^{2}\right)}\right\rangle_{\text {iso }}$ & $6.61 \cdot 10^{-13}$ & $1.21 \cdot 10^{-3}$ & $1.80 \cdot 10^{-2}$ & $3.58 \cdot 10^{-1}$ & 2.23 \\
$\left\langle f_{0 n}^{(\mu O)}\right\rangle_{\text {iso }}$ & $-8.94 \cdot 10^{-7}$ & $-3.23 \cdot 10^{-4}$ & $-4.89 \cdot 10^{-3}$ & $-2.46 \cdot 10^{-1}$ & $-6.03 \cdot 10^{-1}$ \\
$\left\langle f_{0 n}^{(\mu \mathcal{M})}\right\rangle_{\text {iso }}$ & $-9.88 \cdot 10^{-7}$ & $-1.61 \cdot 10^{-3}$ & $-2.40 \cdot 10^{-2}$ & $-4.77 \cdot 10^{-1}$ & -2.97 \\
\hline$\left\langle f_{0 n}^{\left(\mu^{2}\right)}+f_{0 n}^{\left(Q^{2}\right)}+f_{0 n}^{\left(m^{2}\right)}\right\rangle_{\text {iso }}$ & $4.57 \cdot 10^{-4}$ & $2.39 \cdot 10^{-3}$ & $2.94 \cdot 10^{-2}$ & $7.24 \cdot 10^{-1}$ & 3.58 \\
Full $\left\langle f_{0 n}^{(0)}+f_{0 n}^{(2)}\right\rangle_{\text {iso }}$ & $4.55 \cdot 10^{-4}$ & $4.55 \cdot 10^{-4}$ & $4.55 \cdot 10^{-4}$ & $4.55 \cdot 10^{-4}$ & $4.55 \cdot 10^{-4}$ \\
\hline \hline
\end{tabular}


Table III: X-ray absorption oscillator strength for Vinylferrocene, calculated for the lowestenergy transition at the Fe K-edge (excitation energy $7051.3 \mathrm{eV}$ ). The total isotropically averaged oscillator strength and its different contributions are given for different positions of the origin of the coordinate system.

\begin{tabular}{cccccc}
\hline \hline origin & $\boldsymbol{O}$ & $\boldsymbol{O}+\boldsymbol{a}$ & $\boldsymbol{O}+\boldsymbol{a}$ & $\boldsymbol{O}+\boldsymbol{a}$ & $\boldsymbol{O}+\boldsymbol{a}$ \\
& Fe atom & $a_{x}=-100 \AA$ & $a_{z}-100 \AA$ & $a_{x}=-50 \AA$ & $a_{x}=+0.128 \AA$ \\
& & & $a_{z}=-50 \AA$ & $a_{y}=+0.195 \AA$ \\
& & & & $a_{z}=+0.007 \AA$ \\
\hline$\left\langle f_{0 n}^{\left(\mu^{2}\right)}\right\rangle_{\text {iso }}$ & $2.55 \cdot 10^{-6}$ & $2.55 \cdot 10^{-6}$ & $2.55 \cdot 10^{-6}$ & $2.55 \cdot 10^{-6}$ & $2.55 \cdot 10^{-6}$ \\
$\left\langle f_{0 n}^{\left(Q^{2}\right)}\right\rangle_{\text {iso }}$ & $3.09 \cdot 10^{-6}$ & $8.63 \cdot 10^{-2}$ & $7.30 \cdot 10^{-2}$ & $4.47 \cdot 10^{-2}$ & $1.28 \cdot 10^{-6}$ \\
$\left\langle f_{0 n}^{\left(m^{2}\right)}\right\rangle_{\text {iso }}$ & $1.14 \cdot 10^{-12}$ & $2.29 \cdot 10^{-2}$ & $9.04 \cdot 10^{-2}$ & $5.87 \cdot 10^{-3}$ & $1.56 \cdot 10^{-7}$ \\
$\left\langle f_{0 n}^{(\mu O)}\right\rangle_{\text {iso }}$ & $-1.71 \cdot 10^{-8}$ & $-7.83 \cdot 10^{-2}$ & $-4.24 \cdot 10^{-2}$ & $-4.23 \cdot 10^{-2}$ & $6.33 \cdot 10^{-7}$ \\
$\left\langle f_{0 n}^{(\mu \mathcal{M})}\right\rangle_{\text {iso }}$ & $-1.52 \cdot 10^{-8}$ & $-3.09 \cdot 10^{-2}$ & $-1.21 \cdot 10^{-1}$ & $-8.19 \cdot 10^{-3}$ & $9.83 \cdot 10^{-7}$ \\
\hline$\left\langle f_{0 n}^{\left(\mu^{2}\right)}+f_{0 n}^{\left(Q^{2}\right)}+f_{0 n}^{\left(m^{2}\right)}\right\rangle_{\text {iso }}$ & $5.64 \cdot 10^{-6}$ & $1.09 \cdot 10^{-1}$ & $1.63 \cdot 10^{-1}$ & $5.05 \cdot 10^{-2}$ & $3.99 \cdot 10^{-6}$ \\
Full $\left\langle f_{0 n}^{(0)}+f_{0 n}^{(2)}\right\rangle_{\text {iso }}$ & $5.61 \cdot 10^{-6}$ & $5.61 \cdot 10^{-6}$ & $5.61 \cdot 10^{-6}$ & $5.61 \cdot 10^{-6}$ & $5.61 \cdot 10^{-6}$ \\
\hline \hline
\end{tabular}

